\title{
Four-dimensional toric code with non-Clifford transversal gates
}

\author{
Tomas Jochym-O'Connor $\odot^{*}$ and Theodore J. Yoder $\oplus^{\dagger}$ \\ IBM Quantum, IBM T.J. Watson Research Center, Yorktown Heights, New York 10598, USA
}

(Received 15 October 2020; accepted 9 January 2021; published 8 February 2021)

\begin{abstract}
The design of a four-dimensional toric code is explored with the goal of finding a lattice capable of implementing a logical CCCZ gate transversally. The established lattice is the octaplex tessellation, which is a regular tessellation of four-dimensional Euclidean space whose underlying 4-cell is the octaplex, or hyperdiamond. This differs from the conventional $4 \mathrm{D}$ toric code lattice, based on the hypercubic tessellation, which is symmetric with respect to logical $X$ and $Z$ and only allows for the implementation of a transversal Clifford gate. This paper further develops the established connection between topological dimension and transversal gates in the Clifford hierarchy, generalizing the known designs for the implementation of transversal CZ and CCZ in two and three dimensions, respectively.
\end{abstract}

DOI: 10.1103/PhysRevResearch.3.013118

\section{MOTIVATION}

Quantum error correction is expected to play an essential role in the development of large-scale quantum computers. Namely, identifying, controlling, and correcting physical errors will be necessary in running long quantum computations and to that end the theory of quantum fault tolerance has been developed [1]. One of the essential primitives in fault tolerance theory is the notion of a transversal gate, that is, a logical gate that can be implemented by addressing each qubit within a codeblock, individually, in parallel. The primary benefit of such gates is they prevent the propagation of noise between different qubits in the code, which is typically problematic.

Topological error correcting codes are among the most well-studied forms of quantum error correcting codes. These codes are defined by their spatially local stabilizer checks in $D$ dimensions, while embedding their logical information in macroscopic non local degrees of freedom. Such codes provide numerous computing advantages, including a pathway for experimental qubit layout [2-6], efficient decoding algorithms [7-12], and provable target threshold error rates for numerous local noise models $[2,13,14]$. In this paper, we explore the set of transversal gates in one such code, the 4D toric code embedded in the octaplex tessellation. Unlike the symmetric $4 \mathrm{D}$ toric code on the hypercubic lattice, where there is a symmetry between the $X$ and $Z$ stabilizers for the purposes of quantum self-correction [7], the version presented here is asymmetric with respect to $X$ and $Z$ as required by

\footnotetext{
*tjoc@ibm.com

†ted.yoder@ibm.com
}

Published by the American Physical Society under the terms of the Creative Commons Attribution 4.0 International license. Further distribution of this work must maintain attribution to the author(s) and the published article's title, journal citation, and DOI. disjointness [15] to have the ability to implement a nonClifford logical gate transversally.

There is a rich set of literature relating topological codes to logical gates in quantum error correction. Namely, the set of transversal gates accessible to a topological code is related to the spatial dimension in which it is embedded. In the color code family, all Clifford gates are transversal for 2D color codes [16], the 3D color code has a transversal $T$ gate $\left(e^{i \pi Z / 8}\right)$, and more generally $D$-dimensional color codes have transversal $e^{i \pi Z / 2^{D}}$ gates $[17,18]$. It should be noted, while these higher-dimensional codes have more exotic gates, they cannot have both a transversal $e^{i \pi Z / 2^{D}}$ gate with $D>2$ while also having a transversal Hadamard as this would violate nogo theorems for transversal, universal gate sets [19,20].

To better define the relationship between spatial dimension and the transversal gates that are accessible, consider the Clifford hierarchy. The $n$-qubit Clifford hierarchy is defined recursively, where the first level is the $n$-qubit Pauli group $\mathcal{P}_{n}$ :

$$
\mathcal{C}_{n}^{1}=\mathcal{P}_{n}, \mathcal{C}_{n}^{k}=\left\{U \in \mathcal{U}(n): U P U^{\dagger} \in C_{n}^{k-1} \forall P \in P_{n}\right\} .
$$

In general, topological codes in $D$ dimensions will be limited to having transversal gates that are in the $D$ th level of the Clifford hierarchy [21]. The 2D toric code has a transversal controlled- $Z$ (CZ) gate, which is in the second level of the Clifford hierarchy. Moreover, the 3D toric code can be shown to have a transversal controlled-CZ (CCZ) gate, a gate in the third level of the Clifford hierarchy, by finding an explicit mapping between toric and color codes [22]. Recently, Vasmer and Browne gave an explicit transversal CCZ gate with respect to a single-qubit partition for the 3D toric code [23], provided the codes are chosen appropriately. Rather surprisingly, the different codeblocks are not identical, in that one of the codeblocks has stabilizers of different weight than the other two, as will be discussed in Sec. II E.

Given the aforementioned prior work, it is natural to ask whether some choice of 4D toric code has a transversal multiqubit gate in the fourth level of the Clifford hierarchy. In this 
paper, we explore 4D toric codes and show a particular choice of 4D toric code that has an underlying CCCZ gate, a gate in the fourth level of the Clifford hierarchy. The underlying lattice of the code is not a simple generalization of the cubic lattice, such as a hypercubic tessellation, but a rather more exotic lattice that allows for the appropriate overlap conditions of the underlying stabilizers of the four codeblocks.

The paper is organized as follows: Section II is reserved for a review of prior results. In II A, we review the necessary algebraic conditions for CSS codes to have transversal multicontrolled- $Z$ gates. In Sec. II C, we review Schläfli symbols and summarize their relationship to constructing 2D and 3D toric codes in Secs. II D and II E, respectively. In Sec. II F, we present the conditions we will require for searching for a 4D toric code with a transversal CCCZ. Section III provides all the details of the 4D lattice containing a transversal CCCZ, the octaplex tessellation, while Sec. IV discusses placing boundaries on such a lattice. Section $\mathrm{V}$ discusses metachecks and single-shot error correction in the context of this code. Finally, Sec. VI provides a discussion on the difficulty in finding higher-dimensional generalizations and other open questions.

\section{BACKGROUND}

\section{A. Transversal (multi)controlled-Z gates in CSS codes}

Suppose we have multiple codeblocks, each composed of $n$ qubits and containing equal numbers of logical qubits. We denote a Pauli operator $P$ on qubit $i$ of codeblock $c$ as $P_{i}^{(c)}$. We denote an $X$ stabilizer generator of codeblock $c$ as $\mathcal{X}_{i}^{(c)}$, where $i$ is a label for the generator. Similarly, the $Z$ stabilizer generators are labeled as $\mathcal{Z}_{i}^{(c)}$. Logical operators of codeblock $c$ of $X$ type and $Z$ type are represented by $\overline{\mathcal{X}}_{j}^{(c)}$ and $\overline{\mathcal{Z}}_{j}^{(c)}$, respectively. When contextually appropriate, we use $\mathcal{X}_{i}^{(c)}, \overline{\mathcal{X}}_{j}^{(c)}$, etc. to represent the supports of these operators as well.

The controlled- $Z$ (CZ) is a two-qubit Clifford gate that under conjugation maps Pauli $X$ on one qubit to itself times Pauli $Z$ on the other qubit. Mathematically, this relation is expressed as $X_{i}^{(a)} \rightarrow X_{i}^{(a)} Z_{i}^{(b)}$, where the $\mathrm{CZ}$ gate is acting on qubits $i$ of codeblocks $a, b$. We label this a $\mathrm{CZ}_{i}^{(a, b)}$ gate. Additionally, $C Z$ leaves any Pauli $Z$ operator unchanged under conjugation. Therefore, if we have two CSS codeblocks [24,25] of $n$ qubits and we perform a transversal $\mathrm{CZ}$, that is $\prod_{i=1}^{n} \mathrm{CZ}_{i}^{(a, b)}$, then the stabilizer generators are transformed as follows:

$$
\begin{aligned}
& \mathcal{X}_{i}^{(a)}=\prod_{j \in \mathcal{X}_{i}^{(a)}} X_{j}^{(a)} \rightarrow \mathcal{X}_{i}^{(a)} \times \prod_{k \in \mathcal{X}_{i}^{(a)}} Z_{k}^{(b)} \\
& \mathcal{Z}_{i}^{(a)} \rightarrow \mathcal{Z}_{i}^{(a)} .
\end{aligned}
$$

As such, for the codespace to be preserved, we require that the $X$ stabilizers from one codeblock map onto $Z$ stabilizers in the other codeblock. Equivalently, as long as the $X$ stabilizers of a given codeblock overlap an even number of times with the $X$ stabilizers and $X$ logical operators of the other codeblock, the codespace will be preserved. That is, $\forall i, j$,

$$
\begin{aligned}
& \left|\mathcal{X}_{i}^{(a)} \cap \mathcal{X}_{j}^{(b)}\right|=0, \\
& \left|\mathcal{X}_{i}^{(a)} \cap \overline{\mathcal{X}}_{j}^{(b)}\right|=0 .
\end{aligned}
$$

The expression $\mathcal{M} \cap \mathcal{N}$ indicates the overlap in support of two Pauli operators $\mathcal{M}, \mathcal{N}$ while $|\mathcal{O}|$ is the total weight of a given operator $\mathcal{O}$ modulo 2 . Given the above equations are satisfied, transversal CZ will be a logical operator. Yet, for it to implement a logical CZ gate there are additional conditions the logical operators must satisfy: $\forall i, j$,

$$
\left|\overline{\mathcal{X}}_{i}^{(a)} \cap \overline{\mathcal{X}}_{j}^{(b)}\right|=\delta_{i j}
$$

We can generalize these constraints to the controlledcontrolled- $Z$ (CCZ) gate as well. Note that under conjugation, the CCZ gate maps Pauli $X$ on one codeblock onto CZ on the other two codeblocks, that is, $X_{i}^{(a)} \rightarrow X_{i}^{(a)} \mathrm{CZ}_{i}^{(b, c)}$. Additionally, given it is again a diagonal operator CCZ leaves and Pauli $Z$ operator unchanged. The $X$ stabilizer of one codeblock will therefore undergo the following transformation under the action of transversal CCZ:

$$
\mathcal{X}_{i}^{(a)}=\prod_{j \in \mathcal{X}_{i}^{(a)}} X_{j}^{(a)} \rightarrow \mathcal{X}_{i}^{(a)} \times \prod_{k \in \mathcal{X}_{i}^{(a)}} \mathrm{CZ}_{k}^{(b, c)} .
$$

Therefore, to preserve the stabilizer group, we require that $\prod_{k \in \mathcal{X}_{i}^{(a)}} \mathrm{CZ}_{k}^{(b, c)}$ be logical identity, which is equivalent to imposing Eqs. (2), but restricted to the support of the $X$ stabilizer on codeblock $a$. Therefore, the generalized requirement is $\forall i, j, k$,

$$
\begin{aligned}
& \left|\mathcal{X}_{i}^{(a)} \cap \mathcal{X}_{j}^{(b)} \cap \mathcal{X}_{k}^{(c)}\right|=0, \\
& \left|\mathcal{X}_{i}^{(a)} \cap \mathcal{X}_{j}^{(b)} \cap \overline{\mathcal{X}}_{k}^{(c)}\right|=0 \\
& \left|\mathcal{X}_{i}^{(a)} \cap \overline{\mathcal{X}}_{j}^{(b)} \cap \overline{\mathcal{X}}_{k}^{(c)}\right|=0 .
\end{aligned}
$$

Given the above requirements are satisfied, the transversal CCZ maps Pauli $X$ onto a transversal CZ, limited by the support of the Pauli $X$ of the original code. This CZ must in turn perform a logical CZ operation on the other two codes, which impose the additional set of constraints: $\forall i, j, k$,

$$
\left|\overline{\mathcal{X}}_{i}^{(a)} \cap \overline{\mathcal{X}}_{j}^{(b)} \cap \overline{\mathcal{X}}_{k}^{(c)}\right|=[i=j=k],
$$

where we have used the Iverson bracket [26] to denote the overlap being odd when all logical indices are matching and 0 otherwise.

Finally, one can straightforwardly generalize this process to multicontrolled- $Z$ operations. The set of requirements for the transversal CCCZ gate to implement a logical CCCZ on four codeblocks is $\forall i, j, k, l$,

$$
\begin{aligned}
& \left|\mathcal{X}_{i}^{(a)} \cap \mathcal{X}_{j}^{(b)} \cap \mathcal{X}_{k}^{(c)} \cap \mathcal{X}_{l}^{(d)}\right|=0, \\
& \left|\mathcal{X}_{i}^{(a)} \cap \mathcal{X}_{j}^{(b)} \cap \mathcal{X}_{k}^{(c)} \cap \overline{\mathcal{X}}_{l}^{(d)}\right|=0, \\
& \left|\mathcal{X}_{i}^{(a)} \cap \mathcal{X}_{j}^{(b)} \cap \overline{\mathcal{X}}_{k}^{(c)} \cap \overline{\mathcal{X}}_{l}^{(d)}\right|=0, \\
& \left|\mathcal{X}_{i}^{(a)} \cap \overline{\mathcal{X}}_{j}^{(b)} \cap \overline{\mathcal{X}}_{k}^{(c)} \cap \overline{\mathcal{X}}_{l}^{(d)}\right|=0, \\
& \left|\overline{\mathcal{X}}_{i}^{(a)} \cap \overline{\mathcal{X}}_{j}^{(b)} \cap \overline{\mathcal{X}}_{k}^{(c)} \cap \overline{\mathcal{X}}_{l}^{(d)}\right|=[i=j=k=l] .
\end{aligned}
$$

\section{B. Pauli sandwich trick}

One advantage of implementing a CCCZ gate transversally is that if the resulting logical gate couples multiple logical qubits, such as the $3 \mathrm{D}$ toric code with periodic boundaries or 


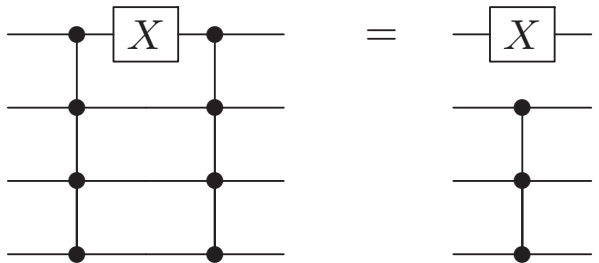

FIG. 1. Pauli sandwich trick: Inserting a Pauli $X$ operator between two CCCZ gates results in a CCZ gate on the other three qubits along with the implemented Pauli $X$. This can be generalized for any multicontrolled- $Z$ operation.

in instances of Pin codes [27], one may still be able to achieve a targeted CCZ gate by repeated uses of the transversal gate. The idea is to insert a logical $X$ gate (which can always be implemented transversally in a stabilizer code) between two instances of the transversal gate implementing logical CCCZ, which results in implementing a CCZ gate on the other three logical codeblocks, see Fig. 1.

For example, as will be shown in this paper, there will be a version of a $4 \mathrm{D}$ toric code with periodic boundaries, encoding four logical qubits, that exhibits a transversal CCCZ. The resulting action of the gate will couple the four logical qubits by implementing four separate logical CCCZ gates across the four codeblocks, see Fig. 2. By sandwiching the appropriate logical $X$ gate we can achieve a transversal implementation of a targeted CCZ logical gate.

\section{Schläfli symbols}

To clarify the upcoming constructions to 4D lattices, we will briefly describe Schläfli symbols and their relationship to regular tessellations. A Schläfli symbol is a succinct description of regular polytopes and tessellations that is defined recursively [28]. Given an integer $n$, the Schläfli symbol $\{n\}$ represents an $n$-sided regular convex polygon. A Schläfli
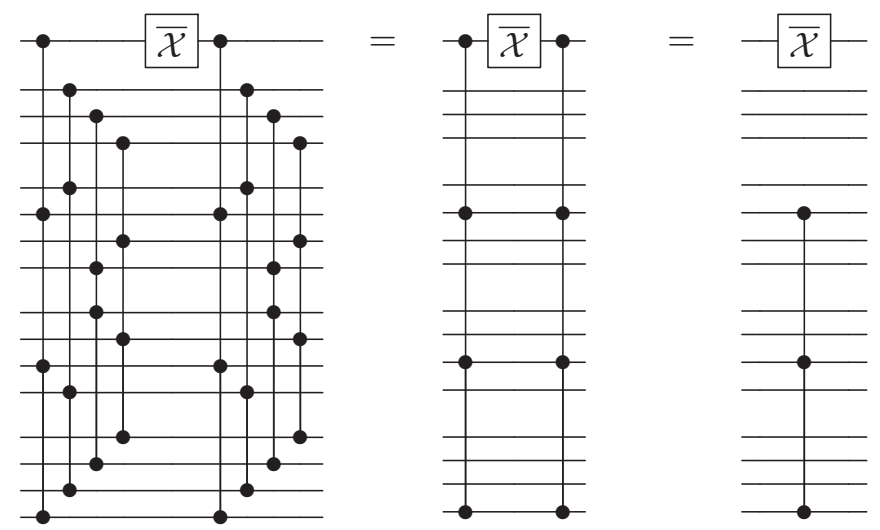

FIG. 2. Assume transversal CCCZ implements the logical gate as shown containing a set of four logical CCCZ gates. Then, by sandwiching a single logical $X$ operator on a given logical qubit between rounds of transversal CCCZ, the resulting logical action is a targeted CCZ. Given any Pauli gate can be implemented transversally in a stabilizer code, the global action also remains transversal.

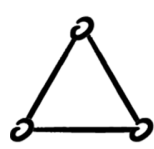

(a)

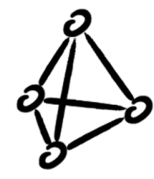

(d)

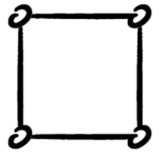

(b)

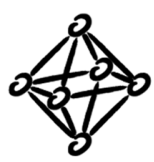

(e)

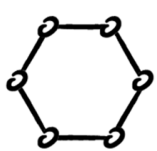

(c)

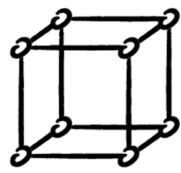

(f)
FIG. 3. A Schläfli symbol $\{n\}$ represents an $n$-sided polygon, while polytopes with Schläfli symbols $\{n, m\}$ have $m n$-sided polygons around each of their vertices. The Schläfli symbols for the various polytopes: (a) $\{3\}$, (b) $\{4\}$, (c) $\{6\}$, (d) $\{3,3\}$, (e) $\{3,4\}$, (f) $\{4,3\}$.

symbol with two integer entries $\{n, m\}$ represents a polytope or tessellation with $m$ symmetrically distributed polytopes $\{n\}$ around each vertex. For example, $\{3,3\}$ represents a tetrahedron as each vertex has three adjacent faces corresponding to equilateral triangles $\{3\}$; see Fig. 3 for further examples.

While it is most straightforward to view Schläfli symbols $\{n, m\}$ as regular convex polytopes in three dimensions, they may also represent tessellations in two-dimensional Euclidean or hyperbolic space. For example, the Schläfli symbol $\{4,4\}$ represents a square tessellation of $2 \mathrm{D}$ space as every vertex is adjacent to four squares, see Fig. 4 for examples. The distinction between these two interpretations can either be made based on context or by use of the words cell/polytope and lattice/tessellation.

In a similar manner, a Schläfli symbol $\{n, m, l\}$ can represent a regular 4-cell or a tessellation of $3 \mathrm{D}$ space, where adjacent to every edge (1-cell) are $l$ polytopes $\{n, m\}$. Importantly for our discussions, the cubic lattice in 3D is described by Schläfli symbol $\{4,3,4\}$ as adjacent to every edge are four cubes $(\{4,3\})$.

Finally, the full generalization of the recursive definition of the Schläfli symbol is as follows: a polytope/tessellation with Schläfli symbol $\left\{r_{1}, \cdots, r_{d}\right\}$ has $r_{d}$ polytopes with Schläfli symbol $\left\{r_{1}, \cdots, r_{d-1}\right\}$ adjacent to every $(d-2)$-cell.

We use the standard definition of the vertex operator of a regular polytope/tessellation to be a polytope centered at

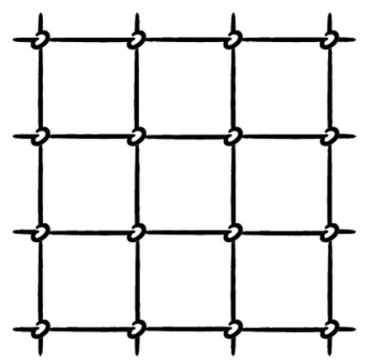

(a)

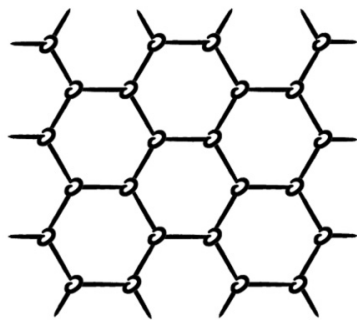

(b)
FIG. 4. Regular tessellations of two-dimensional Euclidean space given by Schläfli symbols $\{n, m\}$. Each vertex is surrounded by $m n$-sided polygons: (a) $\{4,4\}$, (b) $\{6,3\}$. 


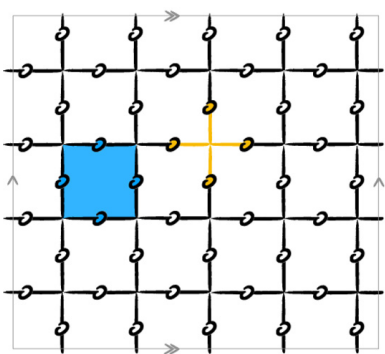

(a)

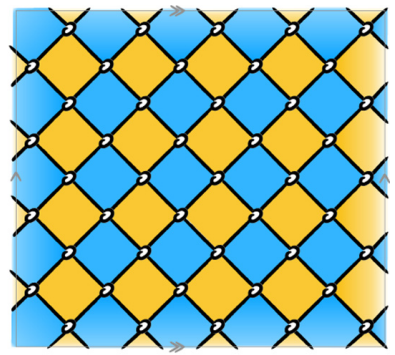

(b)

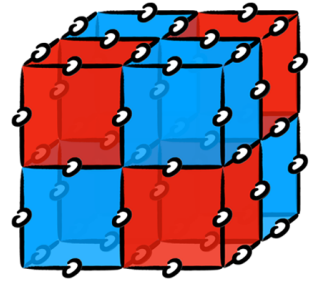

(a)

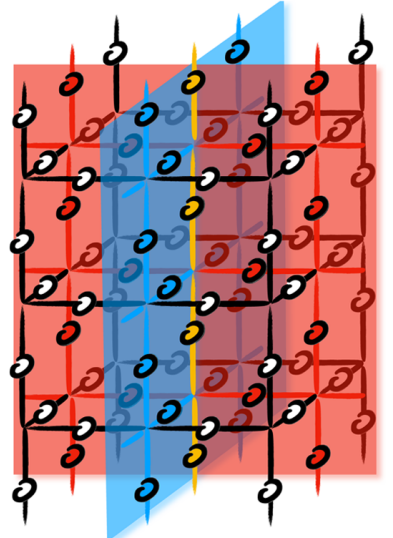

(c)

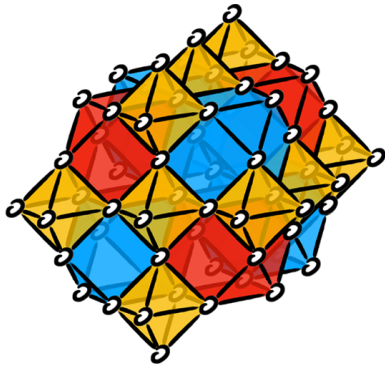

(b)

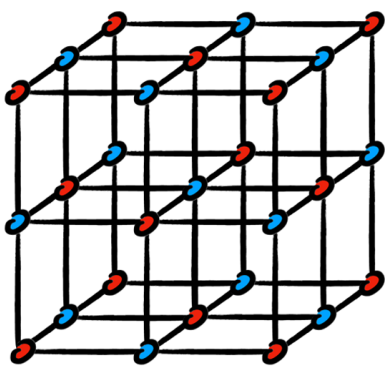

(d) first entry: $\left\{r_{2}, \cdots, r_{d}\right\}$. We shall occasionally also refer to an edge operator which is just the vertex operator of a vertex operator whose Schläfli symbol is determined by removing the first two entries.

\section{2D toric code}

Consider a square lattice $\{4,4\}$ with periodic boundary conditions in both spatial dimensions. The $2 \mathrm{D}$ toric code is defined by placing physical qubits on the edges of the graph and defining the $Z$ stabilizers at the vertices and the $X$ stabilizers on the plaquettes, see Fig. 5 for a visual representation. ${ }^{1}$

Given that the stabilizer code is CSS, we can treat any set of errors by considering separately the individual $X$ and $Z$ components of the error (as the measurement of the stabilizers will project the error onto distinct sets of $X$ and $Z$ errors). Any Pauli string of $X / Z$ errors that does not form a closed loop will violate the stabilizers at the end points of the error string. As such, we say that the errors result in pointlike excitations in the $2 \mathrm{D}$ toric code and the fact they come in pairs as a consequence of the underlying $\mathbb{Z}_{2}$ symmetry. Any closed loop of $X / Z$ errors will commute with the stabilizers and thus return a state in the codespace. If the closed loop is contractible, then it is the product of all stabilizers within the loop, while if it is noncontractible it forms a logical operator.

We can obtain a transversal controlled- $Z$ (CZ) gate between two layers of the 2D toric code if the second copy has swapped the locations of the $X$ and $Z$ stabilizers. It is then straightforward to verify the conditions of Sec. II A as the $X$ stabilizers will clearly overlap an even number of times by

\footnotetext{
${ }^{1}$ The labeling of the $X$ and $Z$ stabilizers are reversed from the traditional definition of the $2 \mathrm{D}$ toric code, as will become evident later in this paper. The choice, however, is equivalent.
}

FIG. 6. Different descriptions of the 3D toric code. (a) Twocoloring of the 3-cells where qubits reside on edges, representing $X$ stabilizers of two codeblocks. $X$ stabilizers of the red (blue) code correspond to qubits supported on individual red (blue) 3-cells. (b) Addition of $X$ stabilizers from third codeblock, in yellow and labeled codeblock 0 , which corresponds to edges sharing the same vertex. (c) $X$ logical operators for first two codeblocks whose intersection forms a $1 \mathrm{D}$ closed loop corresponding to the $Z$ logical operator of codeblock 0. (d) Dual lattice, where physical qubits will reside on 2-cells (faces) and $X$ stabilizers from first two codeblocks will be represented by vertices. Support of the $X$ stabilizers is given by all faces sharing a given vertex.

construction. Moreover, given the switching of the $X$ and $Z$ logical operators between the two code copies, it is clear that logical $X$ from one codeblock will be mapped onto logical $Z$ on the other codeblock, implementing a logical CZ. ${ }^{2}$

\section{E. 3D toric code}

In this subsection, we review the transversal CCZ gate for the 3D toric code due to Vasmer and Browne [23]. Consider the cubic lattice $\{4,3,4\}$ with qubits residing on edges of the lattice. There are three qubits per edge, one for each codeblock. We can then color the cubes in the lattice in two colors, say red and blue, such that cubes of the same color do not share a face (but can share an edge), see Fig. 6(a). Again, we will focus on the case of periodic boundary conditions in all three spatial dimensions; this condition can be relaxed with an appropriate choice of boundaries.

\footnotetext{
${ }^{2}$ Note that for any CSS code we can take a second copy and reverse the roles of $X / Z$ to obtain a transversal $\mathrm{CZ}$.
} 


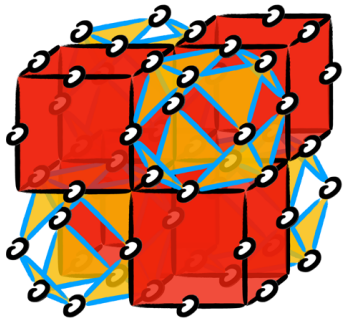

(a)

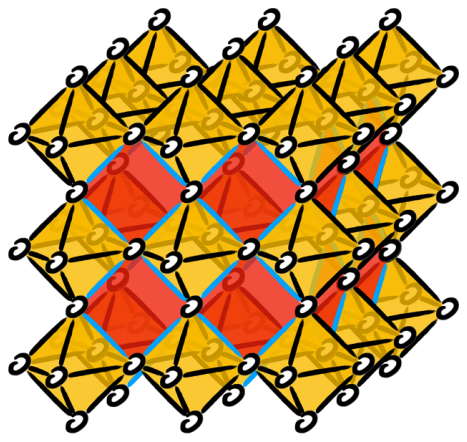

(b)
FIG. 7. $X$ stabilizers and $Z$ stabilizers of the 3D toric code. (a) Codeblock where the $X$ stabilizers are given by red 3-cells, while the $Z$ stabilizers are in intersection of the compliment set of 3 -cells with the vertex operators, given by yellow faces with blue edges. (b) Codeblock where the $X$ stabilizers are given by yellow vertex operators. The $Z$ stabilizers are faces of the intersection of two complimentary 3-cells, shown as red faces with blue edges.

The first codeblock is defined to have $X$ stabilizers supported on the red cubes, while the second codeblock is defined to have $X$ stabilizers supported on the blue cubes. These are weight-12 stabilizers because there are 12 edges to a cube. The third codeblock has weight $6 X$ stabilizers defined by the vertices of the lattice, with the stabilizer supported on neighboring edges, as shown in Fig. 6(b).

Any $Z$ error will give rise to a pair of violated $X$ stabilizers, as in the 2D toric code. As such, a noncontractible loop spanning the lattice forms a logical $Z$ operator. There are three independent logical operators for the $3 \mathrm{D}$ toric code defined on a periodic lattice, one for each dimension. The $X$ logical operator is formed from a 2D plane orthogonal to its conjugate $Z$ logical string pair. See Fig. 6(c) for a pictorial representation of planar $X$ logical operators for the red and blue codeblocks, whose intersection forms a noncontractible loop whose support is that of a $Z$ logical operator for the yellow codeblock.

We can then straightforwardly verify the orthogonality conditions, presented in Sec. II A, for the existence of a transversal CCZ gate. The intersection of $X$ stabilizers from the first two codeblocks corresponds to a weight-4 face belonging to the cubes, such a face will intersect any neighboring vertex operator at the two corresponding adjacent edges of that vertex belonging to the face. Therefore, the intersection of $X$ stabilizers from the three different codeblocks will either be trivial or weight 2 . The $Z$ stabilizers of a codeblock can then be given by all possible intersections of pairs of $X$ stabilizers from the other two codeblocks, see Fig. 7.

The transversal CCZ gate is a logical CCZ gate as described above the intersection of pairs of logical $X$ operators from different codeblocks corresponds to the support of the $Z$ logical operator of the third codeblock, as required.

\section{F. The dual picture}

The dual of a lattice of dimension $D$ is again a $D$ dimensional lattice, where every $D$-cell is replaced by a vertex (0-cell), every $(D-1)$-cell is replaced by an edge (1-cell) connecting two vertices when the corresponding original $D$ cells share the $(D-1)$-cell, and so forth. Conveniently, the dual to any object with Schläfli symbol $\left\{r_{1}, \cdots, r_{d}\right\}$ is the object with Schläfli symbol $\left\{r_{d}, \cdots, r_{1}\right\}$. That is, one reverses the ordering of the integers in the symbol. For example, the dual of a cube $\{4,3\}$ is an octahedron $\{3,4\}$ and the cubic lattice $\{4,3,4\}$ is self-dual.

In the original 2D toric code picture, the $X$ stabilizers are associated to faces, while the $Z$ stabilizers are associated to vertices. The dual of the square lattice is also a square lattice, with faces and vertices interchanged. Therefore, to describe the 2D-toric code in the dual picture, qubits are still placed on edges, but $X$ stabilizers are instead associated to vertices and $Z$ stabilizers to faces.

The 3D-toric codes on the cubic lattice can also be described in the dual picture. The dual of the cubic lattice is again the cubic lattice, but qubits now reside on faces rather than edges. Moreover, $X$ stabilizers of the first two codeblocks are associated with vertices in the dual lattice. The choice of $X$ stabilizers for the two codeblocks is equivalent to a 2-coloring of the vertices in the dual lattice, where vertices of the same color share only faces (never an edge), as shown in Fig. 6(d). The $X$ stabilizers of the third codeblock (originally the vertex operators) are associated with cubes in the dual lattice. The condition, see Eq. (3a), that $X$ stabilizers from the three codeblocks only ever intersect at an even number of qubits can be viewed in the dual picture as any edge (intersection between two vertices corresponding to $X$ stabilizers of codeblocks 1 and 2) sharing only two faces with a given cube.

An additional important property from the $2 \mathrm{D}$ and $3 \mathrm{D}$ toric codes is that the $Z$ logical operator is a noncontractible 1D loop. From the view point of excitations, this arises due to excitations coming in pairs. When a single or connected string of $Z$ errors occur, the only violated syndromes are those at the endpoints of the string, and thus closing the string annihilates the excitations and forms a logical operator. The logical operator is non-trivial (not the product of stabilizers, therefore not the identity operator) if it forms a non-contractible loop. Essential to this reasoning is that $Z$ errors lead to a pair of violated $X$ syndromes, in all codeblocks. Therefore, in the dual picture, any qubit (edge in $2 \mathrm{D}$, face in $3 \mathrm{D}$ ) includes exactly two vertices of the any one color. In $2 \mathrm{D}$, this condition is satisfied trivially, however, in 3D it demands that faces must be squares with opposite corners colored the same.

Therefore, in our quest for regular tessellations in $D$ dimensions that yield interesting multi-controlled- $Z$ gates, we propose the following criteria:

(1) Qubits are placed on edges of the underlying graph. Conversely, qubits are placed on $(D-1)$-cells in the dual lattice.

(2) The vertices of the dual lattice are $(D-1)$-colorable, such that any two vertices of the same color share at most a $(D-1)$-cell

(3) Every $(D-1)$-cell in the dual lattice has two vertices of each of the $(D-1)$ colors.

In this dual-lattice description, qubits correspond to $(D-$ 1 )-cells. The $X$ stabilizers of codeblocks $1, \ldots, D-1$ correspond to vertices of the corresponding colors, $1, \ldots, D-1$. That is, given a vertex, the corresponding $X$-stabilizer is sup- 


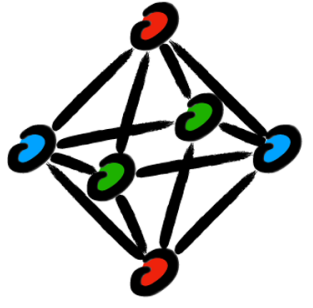

(a)

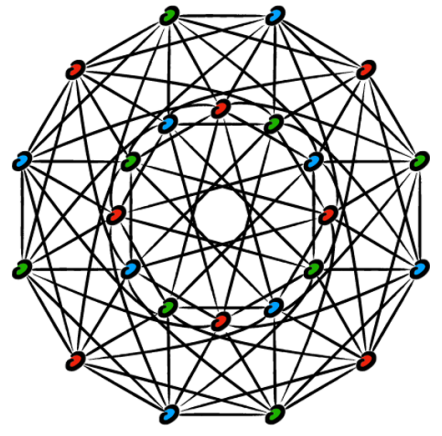

(b)

FIG. 8. The octaplex, Schläfli symbol $\{3,4,3\}$, is composed of 24 vertices which can be three-colored. The vertices in turn compose the 24 octahedra (3-cells), where each edge is surrounded by three octahedra by definition. (a) Three-coloring of an octahedron. (b) Three-coloring of an octaplex.

ported on qubits at all $(D-1)$-cells that contain the vertex. Codeblock 0 is different from the rest in that its $X$ stabilizers are defined by the $D$-cells in the dual lattice, where each $X$ stabilizer is supported on the set of $(D-1)$-cells belonging to a given $D$-cell.

\section{4D CODE WITH TRANSVERSAL CCCZ}

We seek a tessellation of $4 \mathrm{D}$ space with the required desiderata laid out in the previous section. In particular, we search for a lattice which will allow for a set of stabilizers such that the corresponding $Z$ logical operators will be one-dimensional strings and $X$ logical operators will be a three-dimensional hyperplanes. The disparity in the dimension of the logical Pauli operators follows from the disjointness requirements for a transversal gate from the third level of the Clifford hierarchy [15].

We consider the dual lattice, where $X$ stabilizers from the first three codeblocks are given by vertices. Since the lattice should be three-colorable, such that the 3-cells have two vertices of each color, we require the underlying lattice to be composed of octahedra. Therefore, the Schläfli symbol of the tessellation should read $\{3,4, a, b\}$, where $a$ and $b$ are integer degrees of freedom. There is only one such regular tessellation in Euclidean four-space, the octaplex tessellation ${ }^{3}:\{3,4,3,3\}$. In fact, the only other regular tessellations in Euclidean four-space are its dual, the hexadecachoron tessellation, ${ }^{4}\{3,3,4,3\}$, and the tesseractic tessellation, $\{4,3,3,4\}$. In Fig. 8 , we present the threecoloring of the octahedron and the octaplex $\{3,4,3\}$ which form the unit cell for the octaplex tessellation. It should be noted that this lattice was theorized as a potential candidate for the implementation of 4D CCCZ using the theory of Coxeter diagrams [29], which share many of the features of Schläfli symbols. Additionally, note that the vertices of the lattice have

\footnotetext{
${ }^{3}$ Other equivalent names include 24-cell honeycomb and icositetrachoric honeycomb.

${ }^{4}$ Also can be called the 16-cell honeycomb.
}

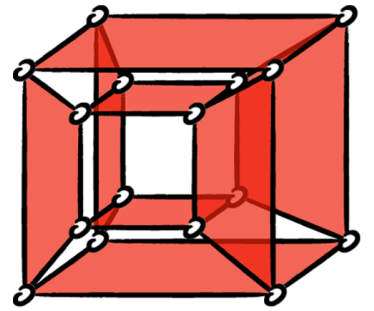

(a)

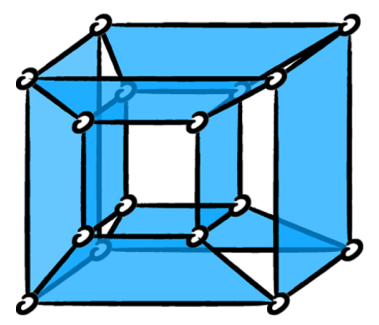

(c)

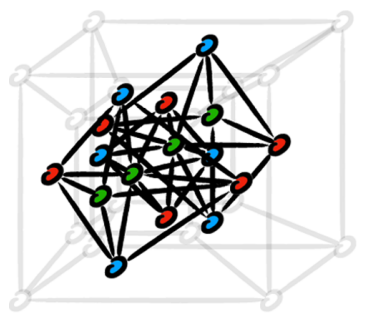

(e)

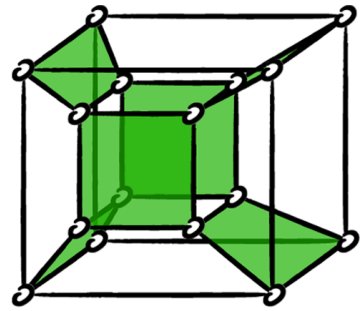

(b)

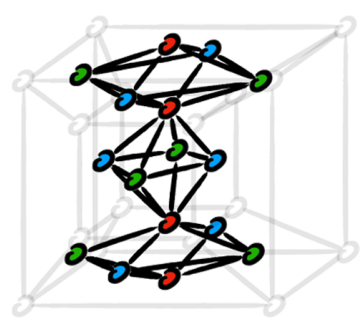

(d)

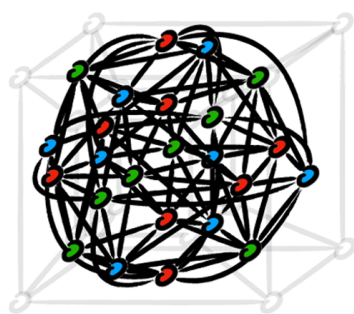

(f)
FIG. 9. Converting between tesseract faces to the orthoplex. (a) Red faces $\mathcal{V}_{r}$. (b) Green faces $\mathcal{V}_{g}$, front and back faces omitted for clarity. (c) Blue faces $\mathcal{V}_{b}$. (d) Subset of vertices of all three colors. (e) Alternative subset of vertices of all three colors. (f) Octaplex!

a one-to-one correspondence with the faces of a tetrahedral lattice, where again the faces can be three-colored such that no faces of the same color share an edge, as shown in Fig. 9

\section{A. Stabilizer weights}

The $X$ stabilizers of codeblock 0 are represented by 4-cells in the 4D tessellation (recall we are working with the dual lattice). That is, each $X$ stabilizer is supported on all 3-cells contained within a 4-cell. By definition, the $4 \mathrm{D}$ tessellation is composed of octaplexes $\{3,4,3\}$. Now, since each octaplex is self-dual, the number of 3-cells within a given octaplex, and the weight of any $X$ stabilizer, is equal to its number of vertices: 24. To determine the weight of the $Z$ stabilizers, we must consider the common intersection of $X$ stabilizers from each of the other codeblocks. Each $X$ stabilizer from the other codeblocks is represented by a different colored vertex and they will only commonly intersect if they form a triangular face in the tessellation. The weight of the $Z$ stabilizer will thus be the number of 3-cells which contain a given face. This can be determined by recursively taking the vertex figure three times. ${ }^{5}$ The resulting geometrical object will have Schläfli

\footnotetext{
${ }^{5}$ Each time one takes a vertex figure, the objects drop in dimension by one. For example, when taking the vertex figure, edges become
} 
symbol $\{3\}$, a triangle, and as such each face is adjacent to three 3 -cells (vertices in the triangle). Therefore, the $Z$ stabilizers will have weight 3.

By construction, codeblocks 1-3 have $X$ stabilizers that are represented by each of the three colors of vertices in the octaplex tessellation. The support of each $X$ stabilizer is the set of 3 -cells containing the given vertex. As such, to calculate the weight of the $X$ stabilizers we can consider the vertex figure: $\{4,3,3\}$, a tesseract. Given the tesseract has 24 faces and each face in the vertex figure represents a 3-cell containing a given vertex, each of the $X$ stabilizers from codeblocks 1-3 are each of weight 24 . The $Z$ stabilizers will be the intersection of an edge (containing two colored vertices) and a 4-cell. That is, we must determine how many 3-cells support a given edge within a given 4-cell. Again this can simply be read off from the Schläfli symbol, as by definition a $\{3,4,3\}$ is the geometric object such that three octahedra $(\{3,4\})$ surround each edge. Therefore, the $Z$ stabilizers for codeblocks 1-3 also have weight 3.

By construction, the fact that codeblock 0 and codeblocks 1-3 each are composed of $X$ stabilizers of weight 24 and $Z$ stabilizer of weight 3 would appear to be coincidental, yet as will become evident in the description in the following subsection and Sec. III E, there is indeed an additional symmetry in the octaplex tessellation that implies that all four codeblocks are equivalent codes.

\section{B. Coordinate system for the octaplex tessellation}

We now give an explicit construction of the octaplex tessellation. First, take the tesseractic tessellation $\{4,3,3,4\}$ on a periodic lattice of size $L$ with integer vertices $(x, y, z, w) \in \mathbb{Z}_{L}^{4}$ and tesseracts centered at half-integer coordinates $\left(x+\frac{1}{2}, y+\right.$ $\left.\frac{1}{2}, z+\frac{1}{2}, w+\frac{1}{2}\right) \in\left(\mathbb{Z}_{L}+\frac{1}{2}\right)^{4}$. The vertices of the octaplex tessellation can be identified with the faces of the tesseractic tessellation, that is coordinates $(x, y, z, w)$ such that two are integers, and two are half-integers. ${ }^{6}$ Vertices share edges if and only if they are distance $1 / \sqrt{2}$ apart in two-norm. We can then label all of the vertices of the octaplex tessellation as one of three colors:

$$
\begin{aligned}
\mathcal{V}_{r}= & \left\{\left(x, y, z+\frac{1}{2}, w+\frac{1}{2}\right) \mid x, y, z, w \in \mathbb{Z}_{L}^{4}\right\} \\
& \cup\left\{\left(x+\frac{1}{2}, y+\frac{1}{2}, z, w\right) \mid x, y, z, w \in \mathbb{Z}_{L}^{4}\right\}, \\
\mathcal{V}_{g}= & \left\{\left(x, y+\frac{1}{2}, z, w+\frac{1}{2}\right) \mid x, y, z, w \in \mathbb{Z}_{L}^{4}\right\} \\
& \cup\left\{\left(x+\frac{1}{2}, y, z+\frac{1}{2}, w\right) \mid x, y, z, w \in \mathbb{Z}_{L}^{4}\right\}, \\
\mathcal{V}_{b}= & \left\{\left(x, y+\frac{1}{2}, z+\frac{1}{2}, w\right) \mid x, y, z, w \in \mathbb{Z}_{L}^{4}\right\} \\
& \cup\left\{\left(x+\frac{1}{2}, y, z, w+\frac{1}{2}\right) \mid x, y, z, w \in \mathbb{Z}_{L}^{4}\right\} .
\end{aligned}
$$

It is straightforward to verify that no two vertices of the same color will share an edge as they will be at least distance 1 away from one another in two-norm.

The 4-cells of the octaplex tessellation are given by coordinates $(x, y, z, w) \in \mathbb{Z}_{L}^{4}$ or $\left(x+\frac{1}{2}, y+\frac{1}{2}, z+\frac{1}{2}, w+\frac{1}{2}\right) \in$

vertices, faces become edges, etc.. Thus, applying it a second time, faces become vertices, 3-cells become edges, etc.

${ }^{6}$ Such faces correspond to the intersection of four tesseracts.
$\left(\mathbb{Z}_{L}+\frac{1}{2}\right)^{4}$ (that is, the same coordinates as the vertices and centers of the hypercubes of the original tesseractic tessellation) and form 24-body objects called octaplexes. The vertices belonging to a octaplex centered at coordinates $(x, y, z, w)$ will be all vertices distance $\frac{1}{\sqrt{2}}$ in two-norm from the corresponding center of the octaplex. For example, for the octaplex centered at the origin, all neighboring vertices will be the set of points $( \pm 1 / 2, \pm 1 / 2,0,0)$ with each \pm taken independently and their permutations, thus totaling a set of 24 vertices. We label the set of 4-cells by $\mathcal{O}$.

The 3-cells correspond to intersections of two neighboring 4-cells, which come in three different types: (3i) the intersection of two 4-cells with integer coordinates differing by \pm 1 in a single coordinate (the center of this type of 4-cell has three integer coordinates and one half-integer), (3ii) the intersection of two 4-cells with half-integer coordinates again differing by \pm 1 in a single coordinate (centered at points with three half-integer and one integer coordinates), or (3iii) the intersection of 4-cells, one with integer and one with half-integer coordinates, differing by $\pm \frac{1}{2}$ in each coordinate (centered at points with four quarter-integer coordinates, that is, points whose entries are odd multiples of $\frac{1}{4}$ ). Each 3-cell contains all vertices that are distance $\frac{1}{2}$ away in two-norm from its center. Given a 3-cell characterized of type (3i), centered say at $\left(x, y, z, w+\frac{1}{2}\right)$ for integers $x, y, z, w$, there are 6 vertices belonging to the 3-cell: $\left(x \pm \frac{1}{2}, y, z, w+\frac{1}{2}\right)$, $\left(x, y \pm \frac{1}{2}, z, w+\frac{1}{2}\right)$, and $\left(x, y, z \pm \frac{1}{2}, w+\frac{1}{2}\right)$. Thus, the 3 -cell is an octahedron with three-coloring as required by Fig. 8(a). A symmetric argument holds for 3-cells of type (3ii). For the 3-cells of type (3iii), the associated vertices are all $\pm \frac{1}{4}$ in each coordinate such that two are integer and half-integer and thus there are $6=\left(\begin{array}{l}4 \\ 2\end{array}\right)$ such coordinates, again forming an octahedron with appropriate coloring as required. We label the set of 3-cells by $\mathcal{Q}$.

It will also be useful to characterize the 2-cells (faces) as they will defined the $Z$ stabilizers for codeblock 0 . As described in Sec. III A, the two-faces are formed from the intersection of three neighboring 2-cells. In fact, each 2-cell will have one neighboring 3-cell of type (3i) or (3ii) and two of type (3iii). Without loss of generality, consider the following 3 -cell of type (3i): $\left(x+\frac{1}{2}, y+\frac{1}{2}, z+\frac{1}{2}, w\right)$ and, in particular, the face whose vertices are $\left\{\left(x+\frac{1}{2}, y+\frac{1}{2}, z, w\right),(x+\right.$ $\left.\left.\frac{1}{2}, y, z+\frac{1}{2}, w\right),\left(x, y+\frac{1}{2}, z+\frac{1}{2}, w\right)\right\}$. Such a face cannot belong to a 3-cell of type (3ii) as any face belonging to such a 3-cell will have to have one of the coordinates being fixed as a half-integer (rather than integer $w$ above). The neighboring 3-cells of type (3iii) will be of the following form: $(x+$ $\left.\frac{1}{4}, y+\frac{1}{4}, z+\frac{1}{4}, w \pm \frac{1}{4}\right)$. Therefore, we label such a 2-cell to be given by the set of coordinates: $\left(x+\frac{1}{4}, y+\frac{1}{4}, z+\frac{1}{4}, w\right){ }^{7}$ Any 2-cell whose coordinates are composed of three quarterintegers and one integer coordinate will be denoted type (2i). Symmetrically, any 2-cell whose coordinates are composed of

\footnotetext{
${ }^{7}$ We note that the chosen labeling does not correspond to the geometric mean of the three vertices belonging to the face. Yet, such a labeling was chosen to simplify notation and can be self-consistent, as explicitly discussed at the end of this section.
} 
three quarter integers and one half-integer coordinate will be labeled as type (2ii).

Finally, it is rather straightforward to verify that 1-cells will always have one integer and a half-integer fixed among the vertices at their endpoints, and will alter between an integer and half-integer in the other two coordinates. As such, the 1cells are all labeled by a set of coordinates composed of one integer, one half-integer, and two quarter-integers.

To summarize, the geometric objects of the octaplex tessellation will be specified by the following forms of cartesian coordinates:

(1) 1-cells (vertices, $\mathcal{V}_{r}, \mathcal{V}_{g}, \mathcal{V}_{b}$ ): Two integer and two half-integer coordinates.

(2) 1-cells: One integer, one half-integer, two quarterintegers.

(3) 2-cells: (2i) One integer and three quarter-integer coordinates. (2ii) One half-integer and three quarter-integer coordinates.

(4) 3-cells (physical qubits, $\mathcal{Q}$ ): (3i) One integer and three half-integer coordinates. (3ii) Three integer and one halfinteger coordinates. (3iii) Four quarter-integer coordinates.

(5) 4-cells $\mathcal{O}$ : (4i) Four integer coordinates. (4ii) Four half-integer coordinates.

An object with dimension $D$ is composed of several objects of dimension $D-1$. In this algebraic construction of the lattice, a $D$-dimensional located at point $P$ is composed of all $(D-1)$-dimensional objects nearest to $P$ in two-norm. We provide more detail in each of the cases.

(1) A 1-cell at coordinate $\left(x, y+\frac{1}{2}, z+\frac{1}{4}, w+\frac{1}{4}\right)$ is composed of two 0 -cells at $\left(x, y+\frac{1}{2}, z+\frac{1}{2}, w\right)$ and at $(x, y+$ $\left.\frac{1}{2}, z, w+\frac{1}{2}\right)$.

(2) (2i) A 2-cell at coordinate $\left(x, y+\frac{1}{4}, z+\frac{1}{4}, w+\frac{1}{4}\right)$ is composed of three 1-cells at $\left(x, y+\frac{1}{2}, z+\frac{1}{4}, w+\frac{1}{4}\right)$, $\left(x, y+\frac{1}{4}, z+\frac{1}{2}, w+\frac{1}{4}\right)$, and $\left(x, y+\frac{1}{4}, z+\frac{1}{4}, w+\frac{1}{2}\right)$. (2ii) A 2-cell at coordinate $\left(x+\frac{1}{2}, y+\frac{1}{4}, z+\frac{1}{4}, w+\frac{1}{4}\right)$ is composed of three 1-cells at $\left(x+\frac{1}{2}, y, z+\frac{1}{4}, w+\frac{1}{4}\right),\left(x+\frac{1}{2}, y+\right.$ $\left.\frac{1}{4}, z, w+\frac{1}{4}\right)$, and $\left(x+\frac{1}{2}, y+\frac{1}{4}, z+\frac{1}{4}, w\right)$.

(3) (3i) A 3-cell at $\left(x, y+\frac{1}{2}, z+\frac{1}{2}, w+\frac{1}{2}\right)$ is composed of 8 2-cells of type $2 \mathrm{i},\left(x, y+\frac{1}{2} \pm \frac{1}{4}, z+\frac{1}{2} \pm \frac{1}{4}, w+\frac{1}{2} \pm \frac{1}{4}\right)$ where each \pm sign can be chosen independently. (3ii) Likewise, a 3-cell at $\left(x+\frac{1}{2}, y, z, w\right)$ is composed of 8 2-cells of type 2ii, $\left(x+\frac{1}{2}, y \pm \frac{1}{4}, z \pm \frac{1}{4}, w \pm \frac{1}{4}\right)$. (3iii) A 3-cell at $P=\left(x+\frac{1}{4}, y+\frac{1}{4}, z+\frac{1}{4}, w+\frac{1}{4}\right)$ is composed of 4 2-cells of each type, located at $P$ plus or minus permutations of the vector $\left(\frac{1}{4}, 0,0,0\right)$.

(4) (4i) A 4-cell at $(x, y, z, w)$ is composed of 24 3 -cells, $\left(x \pm \frac{1}{2}, y, z, w\right), \quad\left(x, y \pm \frac{1}{2}, z, w\right), \quad\left(x, y, z \pm \frac{1}{2}, w\right)$, $\left(x, y, z, w \pm \frac{1}{2}\right)$, and $\left(x \pm \frac{1}{4}, y \pm \frac{1}{4}, z \pm \frac{1}{4}, w \pm \frac{1}{4}\right)$, where again all \pm can be chosen independently. (4ii) Replace $x, y, z$, and $w$ with $x+\frac{1}{2}, y+\frac{1}{2}, z+\frac{1}{2}$, and $w+\frac{1}{2}$ in the (4i) case.

In the language of chain complexes, we have provided a simple description of the boundary operators.

\section{Stabilizer operators of codeblock 0}

The $X$ stabilizers of codeblock 0 are associated to 4-cells, which are given by either integer coordinates $(x, y, z, w) \in \mathbb{Z}_{2}^{4}$ or half integer coordinates $\left(x+\frac{1}{2}, y+\frac{1}{2}, z+\frac{1}{2}, w+\frac{1}{2}\right)$. As discussed in the previous subsection, each of the $X$ stabilizers will be weight- 24 operators whose support is given by the set of 3-cells that are closest in distance from the given 4-cell.

The $Z$ stabilizers are formed from the intersection of the $X$ stabilizers of the other codes. The $X$ stabilizers represented by different colored vertices only have nontrivial intersections if they form a face in the octaplex tessellation. They correspond to weight-3 operators whose support is given by the 3-cells that contain the given face, see the previous subsection for more details.

\section{Logical operators of codeblock 0}

We begin by defining the $Z$ logical operators, which recall are going to be noncontractible loops as per our desiderata. Take the qubit defined by the following 3-cell: $\left(0,0,0, \frac{1}{2}\right)$, which corresponds to the intersection of the two 24-cells $(0,0,0,0)$ and $(0,0,0,1)$. Therefore, a $Z$ error on such a qubit would cause a pair of excitations in the $\hat{w}$ direction, indicating that this qubit should belong to a logical $Z$ operator along that axis. Therefore, the following operator will be a valid logical $Z$ operator,

$$
\overline{\mathcal{Z}}_{\hat{w}}^{(0)}=\prod_{w \in \mathbb{Z}_{L}} Z_{\left(0,0,0, w+\frac{1}{2}\right)}^{(0)},
$$

as it will intersect every $X$ stabilizer $(0,0,0, w)$ at two locations (for all $w$, assuming periodic boundary conditions). While this operator commutes with the stabilizers of the code, what remains to be shown is that it is indeed a logical (nonidentity) Pauli operator, which implies we must be able to find a logical $X$ with which it anticommutes. Before searching for such an operator, note that we can translate the above $Z$ operator by multiplying it by a set of $Z$ stabilizers. As discussed in the last subsection, there is a $Z$ stabilizer supported on qubits in the set:

$$
\begin{aligned}
& \left\{\left(x, y, z, w+\frac{1}{2}\right), \quad\left(x+\frac{1}{4}, y+\frac{1}{4}, z+\frac{1}{4}, w+\frac{1}{4}\right),\right. \\
& \left.\left(x+\frac{1}{4}, y+\frac{1}{4}, z+\frac{1}{4}, w+\frac{3}{4}\right)\right\} .
\end{aligned}
$$

As such, this $Z$ stabilizer will shift any operator supported at $\left(x, y, z, w+\frac{1}{2}\right)$ by $\frac{1}{4}$ in the $\hat{x}, \hat{y}, \hat{z}$ directions while also shifting its support $\pm \frac{1}{4}$ in $\hat{w}$. Note that by choosing different sets of $v_{r}, v_{g}, v_{b}$, we could have also shifted by $-\frac{1}{4}$ in any of the $\hat{x}, \hat{y}, \hat{z}$ directions. We can then continue this shift by multiplying by the $Z$ stabilizer generated by the colored vertices $v_{r}=$ $\left(x+\frac{1}{2}, y+\frac{1}{2}, z, w\right), v_{g}=\left(x+\frac{1}{2}, y, z+\frac{1}{2}, w\right), v_{b}=(x, y+$ $\left.\frac{1}{2}, z+\frac{1}{2}, w\right)$ resulting in the weight- 3 operator:

$\left\{\left(x+\frac{1}{2}, y+\frac{1}{2}, z+\frac{1}{2}, w\right), \quad\left(x+\frac{1}{4}, y+\frac{1}{4}, z+\frac{1}{4}, w-\frac{1}{4}\right)\right.$, $\left.\left(x+\frac{1}{4}, y+\frac{1}{4}, z+\frac{1}{4}, w+\frac{1}{4}\right)\right\}$.

Taking the product of these two weight- 3 operators thus results in a weight- $4 Z$ stabilizer with support:

$$
\begin{aligned}
& \left\{\left(x, y, z, w+\frac{1}{2}\right), \quad\left(x+\frac{1}{4}, y+\frac{1}{4}, z+\frac{1}{4}, w+\frac{3}{4}\right),\right. \\
& \left.\left(x+\frac{1}{4}, y+\frac{1}{4}, z+\frac{1}{4}, w-\frac{1}{4}\right), \quad\left(x+\frac{1}{2}, y+\frac{1}{2}, z+\frac{1}{2}, w\right)\right\},
\end{aligned}
$$

which shifts the logical operator from Eq. (6) as follows:

$$
\begin{aligned}
\overline{\mathcal{Z}}_{\hat{w}}^{(0)} & =\prod_{w \in \mathbb{Z}_{L}} Z_{\left(0,0,0, w+\frac{1}{2}\right)}^{(0)} \\
& \simeq \prod_{w \in \mathbb{Z}_{L}} Z_{\left(\frac{1}{4}, \frac{1}{4}, \frac{1}{4}, w+\frac{1}{4}\right)}^{(0)} Z_{\left(\frac{1}{4}, \frac{1}{4}, \frac{1}{4}, w+\frac{3}{4}\right)}^{(0)}
\end{aligned}
$$




$$
\begin{aligned}
& \simeq \prod_{w \in \mathbb{Z}_{L}} Z_{\left(\frac{1}{4}, \frac{1}{4}, \frac{1}{4}, w-\frac{1}{4}\right)}^{(0)} Z_{\left(\frac{1}{2}, \frac{1}{2}, \frac{1}{2}, w\right)}^{(0)} Z_{\left(\frac{1}{4}, \frac{1}{4}, \frac{1}{4}, w+\frac{3}{4}\right)}^{(0)} \\
& =\prod_{w \in \mathbb{Z}_{L}} Z_{\left(\frac{1}{2}, \frac{1}{2}, \frac{1}{2}, w\right)}^{(0)},
\end{aligned}
$$

where the terms given by coordinates $\left(\frac{1}{4}, \frac{1}{4}, \frac{1}{4}, w-\frac{1}{4}\right)$ and $\left(\frac{1}{4}, \frac{1}{4}, \frac{1}{4}, w+\frac{3}{4}\right)$ cancel each other out given periodic boundary conditions. It is worth pointing out that by choosing a different set of $Z$ stabilizers we could have shifted the above logical operator $\pm \frac{1}{2}$ in any $\hat{x}, \hat{y}, \hat{z}$ direction and as such by iterating this process we can show that all of the following representations are equivalent:

$$
\begin{aligned}
& \overline{\mathcal{Z}}_{\hat{w}}^{(0)}=\prod_{w \in \mathbb{Z}_{L}} Z_{\left(0,0,0, w+\frac{1}{2}\right)}^{(0)} \\
& \simeq \prod_{w \in \mathbb{Z}_{L}} Z_{\left(x, y, z, w+\frac{1}{2}\right)}^{(0)}
\end{aligned}
$$

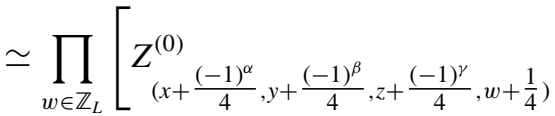

$$
\begin{aligned}
& \left.\times Z^{(0)}\left(x+\frac{(-1)^{\alpha}}{4}, y+\frac{(-1)^{\beta}}{4}, z+\frac{(-1)^{\gamma}}{4}, w+\frac{3}{4}\right)\right] \\
& \simeq \prod_{w \in \mathbb{Z}_{L}} Z_{\left(x+\frac{1}{2}, y+\frac{1}{2}, z+\frac{1}{2}, w\right)}^{(0)}
\end{aligned}
$$

for all $x, y, z \in \mathbb{Z}_{L}$ and $\alpha, \beta, \gamma \in \mathbb{Z}_{2}$. Therefore, given that the $Z$ logical operator can be shifted in any $\hat{x}, \hat{y}, \hat{z}$ direction the corresponding $X$ logical operator will have to span these three axes, thus forming a hyperplane. This is analogous to the $X$ logical operator spanning a plane orthogonal to the $Z$ loop operator in the $3 \mathrm{D}$ toric code. In fact, by the above observation that we can find three disjoint representatives according to the $\hat{w}$ coordinate being either an integer, half-integer, or quarter-integer, the corresponding $X$ logical operator will be composed of three hyperplanes with a fixed $\hat{w}$ coordinate of each of these three types. We propose the following $X$ logical operator to be that which is orthogonal to $\overline{\mathcal{Z}}_{\hat{w}}^{(0)}$ :

$$
\begin{array}{r}
\overline{\mathcal{X}}_{\hat{w}}^{(0)}=\prod_{\substack{x, y, z \\
\in \mathbb{Z}_{L}}}\left[\prod_{\substack{\alpha, \beta, \gamma \\
\in\{0,1\}}} X_{\left(x, y, z, \frac{1}{2}\right)}^{(0)} X_{\left(x+\frac{1}{2}, y+\frac{1}{2}, z+\frac{1}{2}, 0\right)}^{(0)}\right. \\
\left.\times X_{\left(x+\frac{(-1)^{\alpha}}{4}, y+\frac{(-1)^{\beta}}{4}, z+\frac{(-1)^{\gamma}}{4}, \frac{1}{4}\right)}^{(0)}\right] .
\end{array}
$$

We leave the proof that this operator commutes with the stabilizer group, and is thus a logical operator, to Appendix A. It is straightforward to verify that it intersects the logical $Z$ representative from Eq. (6) at a single qubit given by coordinate $\left(0,0,0, \frac{1}{2}\right)$, and as such the two operator anticommute. Finally, given codeblock 0 is symmetric with respect to all four spatial directions, we can define the other three pairs of logical operators as follows:

$$
\overline{\mathcal{Z}}_{\hat{x}}^{(0)}=\prod_{x \in \mathbb{Z}_{L}} Z_{\left(x+\frac{1}{2}, 0,0,0\right)}^{(0)},
$$$$
\overline{\mathcal{X}}_{\hat{x}}^{(0)}=\prod_{\substack{y, z, w \\ \in \mathbb{Z}_{L}}}\left[\prod_{\substack{\beta, \gamma, \zeta \\ \in\{0,1\}}} X_{\left(\frac{1}{2}, y, z, w\right)}^{(0)} X_{\left(0, y+\frac{1}{2}, z+\frac{1}{2}, w+\frac{1}{2}\right)}^{(0)}\right.
$$$$
\left.\times X_{\left(\frac{1}{4}, y+\frac{(-1)^{\beta}}{4}, z+\frac{(-1)^{\gamma}}{4}, w+\frac{(-1)^{\zeta}}{4}\right)}^{(0)} \prod_{\substack{\alpha, \beta, \gamma \\ \in\{0,1\}}}\right],
$$$$
\overline{\mathcal{Z}}_{\hat{y}}^{(0)}=\prod_{y \in \mathbb{Z}_{L}} Z_{\left(0, y+\frac{1}{2}, 0,0\right)}^{(0)},
$$$$
\overline{\mathcal{X}}_{\hat{y}}^{(0)}=\prod_{\substack{x, z, w \\ \in \mathbb{Z}_{L}}}\left[\prod_{\substack{\alpha, \gamma, \zeta \\ \in\{0,1\}}} X_{\left(x, \frac{1}{2}, z, w\right)}^{(0)} X_{\left(x+\frac{1}{2}, 0, z+\frac{1}{2}, w+\frac{1}{2}\right)}^{(0)}\right.
$$

$$
\begin{aligned}
& \left.\times X_{\left(x+\frac{(-1)^{\alpha}}{4}, \frac{1}{4}, z+\frac{(-1)^{\gamma}}{4}, w+\frac{(-1)^{\zeta}}{4}\right)}^{(0)} \prod_{\substack{\alpha, \beta, \gamma \\
\in\{0,1\}}}\right], \\
& \overline{\mathcal{Z}}_{\hat{z}}^{(0)}=\prod_{z \in \mathbb{Z}_{L}} Z_{\left(0,0, z+\frac{1}{2}, 0\right)}^{(0)}, \\
& \overline{\mathcal{X}}_{\hat{z}}^{(0)}=\prod_{\substack{x, y, w \\
\in \mathbb{Z}_{L}}}\left[\prod_{\substack{\alpha, \beta, \zeta \\
\in\{0,1\}}} X_{\left(x, y, \frac{1}{2}, w\right)}^{(0)} X_{\left(x+\frac{1}{2}, y+\frac{1}{2}, 0, w+\frac{1}{2}\right)}^{(0)}\right.
\end{aligned}
$$

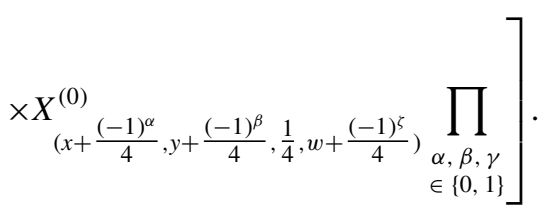

\section{E. Equivalence of all four codeblocks}

Recall the other codeblocks are defined by $X$ stabilizers that are supported on the vertices of a given color. To show equivalence between codeblocks 0 and 1, suppose we introduce new vertices where all of the 4-cells are centered, while eliminating all of the red vertices and introducing edges following the same rules as in Sec. III B. The new set of vertices will be

$$
\begin{aligned}
\mathcal{V}_{r}^{\prime}= & \left\{(x, y, z, w) \mid x, y, z, w \in \mathbb{Z}_{L}^{4}\right\} \\
& \cup\left\{\left(x+\frac{1}{2}, y+\frac{1}{2}, z+\frac{1}{2}, w+\frac{1}{2}\right) \mid x, y, z, w \in \mathbb{Z}_{L}^{4}\right\}, \\
\mathcal{V}_{g}^{\prime}= & \left\{\left(x, y+\frac{1}{2}, z, w+\frac{1}{2}\right) \mid x, y, z, w \in \mathbb{Z}_{L}^{4}\right\} \\
& \cup\left\{\left(x+\frac{1}{2}, y, z+\frac{1}{2}, w\right) \mid x, y, z, w \in \mathbb{Z}_{L}^{4}\right\}, \\
\mathcal{V}_{b}^{\prime}= & \left\{\left(x, y+\frac{1}{2}, z+\frac{1}{2}, w\right) \mid x, y, z, w \in \mathbb{Z}_{L}^{4}\right\} \\
& \cup\left\{\left(x+\frac{1}{2}, y, z, w+\frac{1}{2}\right) \mid x, y, z, w \in \mathbb{Z}_{L}^{4}\right\},
\end{aligned}
$$


where vertices share an edge if they are distance $\frac{1}{\sqrt{2}}$ in twonorm and we define 4-cells centered where all of the old red vertices were located $\mathcal{V}_{r}$, where each 4-cell contains all vertices again at distance $\frac{1}{\sqrt{2}}$ in two-norm. If we then introduce the change of coordinates: $(x, y, z, w) \rightarrow\left(x+\frac{1}{2}, y+\right.$ $\left.\frac{1}{2}, z, w\right)$, we note we have the exact same lattice as previously where the rolls of different sets of vertices and 4-cells have been exchanged:

$$
\begin{aligned}
\mathcal{V}_{r} & \rightarrow \mathcal{O}, \\
\mathcal{V}_{g} & \rightarrow \mathcal{V}_{b}, \\
\mathcal{V}_{b} & \rightarrow \mathcal{V}_{g}, \\
\mathcal{O} & \rightarrow \mathcal{V}_{r}, \\
\mathcal{Q} & \rightarrow \mathcal{Q} .
\end{aligned}
$$

Thus, under this new labeling, codeblock 1 has $X$ stabilizers that were previously labeled by the red vertices and are now defined by 4-cells, with $Z$ stabilizers defined by the intersection of different colored vertices in the new labeling. As such, the properties of codeblock 1 mirror those of codeblock 0 . Given our choice of which colored vertices to eliminate was arbitrary all codeblocks are indeed symmetric.

Given the symmetry of the codeblocks, we can use the above change of basis to determine the logical operators for the other codeblocks as well. For example, the logical $\overline{\mathcal{Z}}_{\hat{w}}^{(1)}$ (red codeblock) will be a shifted version of that from codeblock 0:

$$
\begin{aligned}
& \overline{\mathcal{Z}}_{\hat{w}}^{(0)}=\prod_{w} Z_{\left(0,0,0, w+\frac{1}{2}\right)}^{(0)} \quad \longrightarrow \quad \overline{\mathcal{Z}}_{\hat{w}}^{(1)}=\prod_{w} Z_{\left(\frac{1}{2}, \frac{1}{2}, 0, w+\frac{1}{2}\right)}^{(1)} \\
& \simeq \prod_{w} Z_{\left(-\frac{1}{2},-\frac{1}{2}, \frac{1}{2}, w\right)}^{(0)} \quad \longrightarrow \quad \simeq \prod_{w} Z_{\left(0,0, \frac{1}{2}, w\right) .}^{(1)}
\end{aligned}
$$

We can find the Pauli logical operators for all other codeblocks in a systematic way by modifying the change of coordinates; we list them all in Appendix C.

In a similar manner, we can establish the logical $X$ operator of the other codeblocks by imposing the appropriate shift of coordinates. For example, for codeblock 1:

$$
\begin{gathered}
\overline{\mathcal{X}}_{\hat{w}}^{(0)}=\prod_{\substack{x, y, z \\
\in \mathbb{Z}_{L}}}\left[\prod_{\substack{\alpha, \beta, \gamma \\
\in\{0,1\}}} X_{\left(x, y, z, \frac{1}{2}\right)}^{(0)} X_{\left(x+\frac{1}{2}, y+\frac{1}{2}, z+\frac{1}{2}, 0\right)}^{(0)}\right. \\
\left.\times X_{\left(x+\frac{(-1)^{\alpha}}{4}, y+\frac{(-1)^{\beta}}{4}, z+\frac{(-1)^{\gamma}}{4}, \frac{1}{4}\right)}^{(0)}\right] \\
\longrightarrow \prod_{\substack{x, y, z \\
\in \mathbb{Z}_{L}}}\left[\prod_{\substack{\alpha, \beta, \gamma \\
\in\{0,1\}}} X_{\left(x+\frac{1}{2}, y+\frac{1}{2}, z, \frac{1}{2}\right)}^{(1)} X_{\left(x+1, y+1, z+\frac{1}{2}, 0\right)}^{(1)}\right.
\end{gathered}
$$

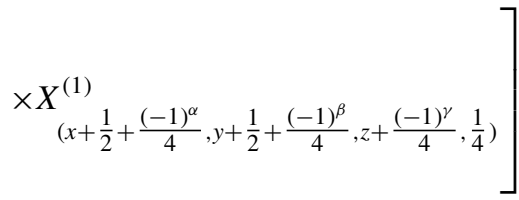

$$
\begin{aligned}
& \simeq \prod_{\substack{x, y, z \\
\in \mathbb{Z}_{L}}}\left[\prod_{\substack{\alpha, \beta, \gamma \\
\in\{0,1\}}} X_{\left(x+\frac{1}{2}, y+\frac{1}{2}, z, \frac{1}{2}\right)}^{(1)} X_{\left(x, y, z+\frac{1}{2}, 0\right)}^{(1)}\right. \\
& \left.\times X_{\left(x+\frac{(-1)^{\alpha}}{4}, y+\frac{(-1)^{\beta}}{4}, z+\frac{(-1)^{\gamma}}{4}, \frac{1}{4}\right)}^{(1)}\right]=\overline{\mathcal{X}}_{\hat{w}}^{(1)} .
\end{aligned}
$$

\section{F. Transversal CCCZ gate}

Given the code construction, transversal CCCZ results in a logical operator. We now verify that it indeed implements the logical CCCZ across the four logical qubits. A detailed proof that the given logical operators satisfy the criteria established in Sec. II A is given in Appendix C, yet we summarize the result here.

As presented in the Sec. III D, each of the logical $Z$ operators can be represented by noncontractible strings in each of the Cartesian directions. Each corresponding logical $X$ is a hyperplane orthogonal to the direction of the logical $Z$. As such, when considering the overlap of different logical $X$ operators from different codeblocks, those that have nontrivial overlap span different directions. The intersection of two hyperplanes that are not parallel is a two-dimensional plane. Taking the intersection with yet another orthogonal hyperplane gives rise to a $1 D$ string. Therefore, the intersection of three nonparallel logical $X$ operators is a $1 \mathrm{D}$ noncontractible string in the fourth codeblock, which corresponds exactly to the support of the fourth logical $Z$ operator on that code, as required for the transversality of the logical CCCZ.

Transversal CCCZ thus results in a logical CCCZ that couples the following quartets of labeling the logical operators:

$$
\begin{aligned}
& \left\{\overline{\mathcal{X}}_{\hat{w}}^{(0)}, \overline{\mathcal{X}}_{\hat{z}}^{(1)}, \overline{\mathcal{X}}_{\hat{y}}^{(2)}, \overline{\mathcal{X}}_{\hat{x}}^{(3)}\right\}, \\
& \left\{\overline{\mathcal{X}}_{\hat{z}}^{(0)}, \overline{\mathcal{X}}_{\hat{w}}^{(1)}, \overline{\mathcal{X}}_{\hat{x}}^{(2)}, \overline{\mathcal{X}}_{\hat{y}}^{(3)}\right\}, \\
& \left\{\overline{\mathcal{X}}_{\hat{y}}^{(0)}, \overline{\mathcal{X}}_{\hat{x}}^{(1)}, \overline{\mathcal{X}}_{\hat{w}}^{(2)}, \overline{\mathcal{X}}_{\hat{z}}^{(3)}\right\}, \\
& \left\{\overline{\mathcal{X}}_{\hat{x}}^{(0)}, \overline{\mathcal{X}}_{\hat{y}}^{(1)}, \overline{\mathcal{X}}_{\hat{z}}^{(2)}, \overline{\mathcal{X}}_{\hat{w}}^{(3)}\right\} .
\end{aligned}
$$

Note that rather than logical operators with the same index being coupled, it is those with different indices that are coupled. This reflects that the logical operators must span directions that are orthogonal from one another. In terms of the criteria in Eq. (4e), the right side of the equality would be 1 if and only if the indices on the left come from one of the sets above.

\section{BOUNDARY CONDITIONS}

Thus far, to simplify the discussion, we have presented a code construction with periodic boundary conditions, encoding four logical qubits across the four codeblocks. Moreover, 


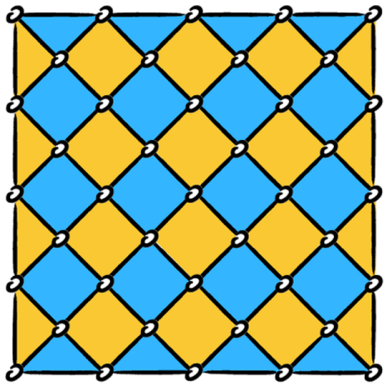

(a)

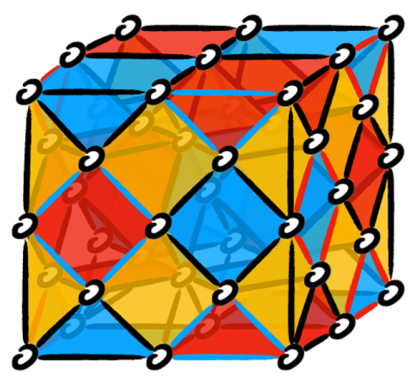

(b)
FIG. 10. (a) 2D toric code with boundaries. (b) 3D toric code with boundaries.

as in the $2 \mathrm{D}$ and $3 \mathrm{D}$ toric codes, we can also introduce boundary conditions such that the code does not have to be defined on a periodic lattice at the cost of now only encoding a single logical qubit. ${ }^{8}$

As first introduced in the 2D toric code [30], we can introduce two types of boundaries, rough and smooth, that can serve as endpoints for the different types of logical Pauli operators $X$ and $Z$, respectively. As discussed in the previous section, to have a transversal logical $C C C Z$ gate on the single logical qubit, we require that the logical $Z$ operators of the four codeblocks span orthogonal axes. As such, the associated rough boundaries should be hyperplanes that are each orthogonal to the respective logical $Z$ operators in each code. The smooth boundaries in each of the codeblocks should be hyperplanes orthogonal to the other three axes. This is analogous to the case of the $2 \mathrm{D}$ and $3 \mathrm{D}$ toric codes with boundaries presented in Fig. 10.

The main idea for constructing the code with boundaries is to begin with the periodic case and remove hyperplanes of qubits, thereby cutting the lattice along each of the Cartesian coordinates. As such, we remove a set of $X$ stabilizers along the hyperplane cut and modify any $X$ stabilizers at the boundary to have slightly smaller support (analogous to both the 2D and 3D cases). Given a CSS code can be defined just in terms of its $X$ stabilizers and logical operators (with the $Z$ stabilizers derivable from these alone), we define these sets for the four codeblocks and determine the associated $Z$ stabilizers and logical operators, where there is a single logical operator per codeblock.

We begin with codeblock 0 , that is, the codeblock where $X$ stabilizers are defined by four integer $(x, y, z, w)$ or four half-integer $\left(x+\frac{1}{2}, y+\frac{1}{2}, z+\frac{1}{2}, w+\frac{1}{2}\right)$ coordinates, where previously we were working with periodic coordinates in $\mathbb{Z}_{L}$. We want to choose boundary conditions such that the logical $Z$ operator defined in Eq. (6) has a $\hat{w}$ coordinate that runs between $\left[\frac{1}{2}, L\right]$. Therefore, we must choose $X$ stabilizers $(x, y, z, w),\left(x+\frac{1}{2}, y+\frac{1}{2}, z+\frac{1}{2}, w+\frac{1}{2}\right)$ such that $w \in$ $[1, L-1]$. Therefore, we remove all qubits whose support in the $\hat{w}$ coordinate falls outside the interval $\left[\frac{1}{2}, L\right]$.

\footnotetext{
${ }^{8}$ In this paper, we omit the cases of introducing holes or twists in the lattice to increase the number of logical qubits.
}

By the symmetry arguments of Sec. IIIE, similar qubits are removed along the other axes as well, thus removing any qubits with a coordinate outside the aforementioned set $[1, L]$. The associated $Z$ logical operator has the following support:

$$
\begin{aligned}
\overline{\mathcal{Z}}_{\hat{w}}^{(0)} & =\prod_{w=1}^{L} Z_{\left(x, y, z, w-\frac{1}{2}\right)}^{(0)} \\
& \simeq \prod_{w=1}^{L} Z_{\left(x-\frac{1}{2}, y-\frac{1}{2}, z-\frac{1}{2}, w\right)}^{(0)},
\end{aligned}
$$

where $x, y, z$ are any integers in the set $[1, L]$. The corresponding logical $X$ operator is

$$
\begin{aligned}
\overline{\mathcal{X}}_{\hat{w}}^{(0)}=\prod_{x, y, z=1}^{L} & {\left[\prod_{\substack{\alpha, \beta, \gamma \\
\in\{0,1\}}} X_{\left(x, y, z, w-\frac{1}{2}\right)}^{(0)} X_{\left(x-\frac{1}{2}, y-\frac{1}{2}, z-\frac{1}{2}, w\right)}^{(0)}\right.} \\
& \left.\times X_{\left(x+\frac{(-1)^{\alpha}}{4}, y+\frac{(-1)^{\beta}}{4}, z+\frac{(-1)^{\gamma}}{4}, w-\frac{1}{4}\right)}^{(0)}\right] .
\end{aligned}
$$

Now, it should be noted that while we only preserve the $X$ stabilizers whose $\hat{w}$ coordinate is in the interval $\left[1, L-\frac{1}{2}\right]$, in the other coordinate directions we will also include those stabilizers whose coordinates are in the set $\left\{\frac{1}{2}, L\right\}$ and such stabilizers will have smaller support as some of their original qubits have been removed. This is analogous to the boundary stabilizers in the 2D and 3D toric codes, whose support is also smaller than in the original periodic lattice. Moreover, it is the addition of these stabilizers that prevent the boundaries along these axes from being smooth, and thus preventing a logical $Z$ operator from terminating there. For example, consider the stabilizer whose coordinates are $\left(\frac{1}{2}, y+\frac{1}{2}, z+\frac{1}{2}, w+\frac{1}{2}\right)$, then the analogous logical operator $\overline{\mathcal{Z}}_{\hat{x}}^{(0)}$, in the $\hat{x}$ direction, would not commute with the example stabilizer as they would intersect at only one qubit: $\left(1, y+\frac{1}{2}, z+\frac{1}{2}, w+\frac{1}{2}\right)$. See Appendix $\mathrm{F}$ for a summary of the stabilizers at the boundary and their support.

The $Z$ stabilizers of a given codeblock are formed by taking the intersection of the $X$ stabilizers of the other three codeblocks, thus preserving the requirements for transversal $\mathrm{CCCZ}$

\section{COUNTING STABILIZERS: METACHECKS AND SINGLE-SHOT $Z$ STABILIZER MEASUREMENT}

In this section, we count the degrees of freedom in the code and show that there are indeed only four logical qubits in the 4D octaplex tessellation. This is due to the high amount of redundancy in the $Z$ stabilizer checks. We refer to these redundancies as metachecks following the language of Ref. [31].

Given we have established a symmetry between all of the codeblocks (see Sec. IIIE), we focus on codeblock 0 as on a periodic lattice. Physical qubits are given by the following coordinates found in Sec. IIIB: (3i) one integer and three half-integers, (3ii) three integer and one half-integer, and (3iii) 


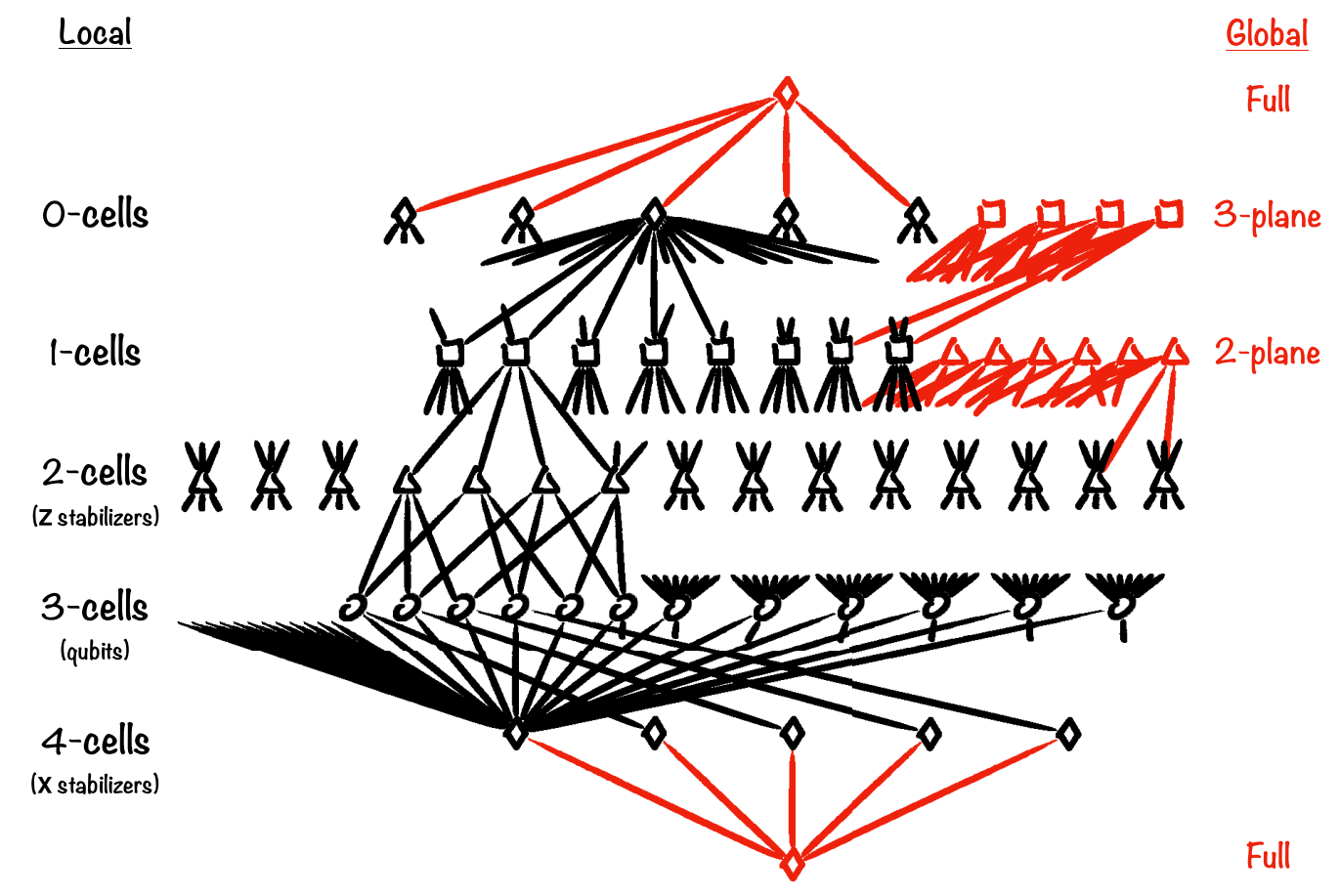

FIG. 11. Extended Tanner graph description of the octaplex tessellation. Physical qubits are associated with 3-cells. In codeblock 0, $X$ stabilizers are associated with 4-cells where the 3-cells present in a given 4-cell indicate the support of a given $X$ stabilizer. $Z$ stabilizers are associated with 2-cells, which are triangles, whose supports are given by all 3-cells sharing a given face. Not all $X$ and $Z$ stabilizers are independent and are related through metachecks given by the 0 - and 1-cells, local symmetry checks, as well as checks corresponding to symmetries that span the full lattice, called global symmetry checks, given in red.

four quarter-integers. Therefore, the total number of physical qubits is $8 L^{4}+(2 L)^{4}=24 L^{4}$.

In codeblock 0 , the $X$ stabilizers are associated with the 4-cells of the tessellation, which have coordinates that are either all integer or half-integer. As such, the number of such stabilizers is $2 L^{4}$ and their product is identity, yielding a independent generator set of size $2 L^{4}-1$. We call this redundancy in the stabilizer generators a global metacheck as it corresponds to a global symmetry of the lattice. The redundancy of one of the $X$ stabilizers is represented by the bottom metacheck in Fig. 11 relating all 4-cells.

Recall the $Z$ stabilizers of codeblock 0 are identified with by the triangular faces (with three different colored vertices) in the octaplex tessellation. Therefore, to count the number of such faces it is sufficient to count the associated faces to which a single vertex belongs to, which amounts to determining the number of edges in the vertex operator (see Sec. II C for a review of vertex operators). The vertex operator of the octaplex tessellation has Schläfli symbol $\{4,3,3\}$, a tesseract, and as such has 32 edges. The number of vertices of a given color is $2 L^{4}$ and as such the number of $Z$ stabilizers corresponding to triangular faces is $64 L^{4}$. Of course, these stabilizers are not all independent and we shall review their dependencies here.

Each 2-cell is a face belonging to three adjacent 3-cells, which corresponds to the qubit support of the Pauli operator defined on the 2-cell. Therefore, a set of 2-cells is said to be dependent if their overlap is even across all 3-cells. There are two forms of such symmetries for the 2-cells: local symmetries which are the result of a product of local faces that result in the identity operator, or global symmetries which result from a product of faces that span the lattice that result in identity.

We begin with the local symmetry: Consider an edge in the octaplex tessellation and the associated triangular faces that contain the given edge. There are four such faces as the associated edge operator ${ }^{9}$ is a tetrahedron $\{3,3\}$, where the vertices of the tetrahedron represent neighboring faces and the edges represent neighboring 3-cells. Each neighboring 3 -cell is adjacent to two such faces and as such the product over all such faces yields the identity. ${ }^{10}$ Therefore, each edge in the octaplex tessellation provides a redundancy in the $Z$ stabilizers. Each vertex belongs to 16 edges (number of vertices in the vertex operator) and as such given the total number of vertices is $6 L^{4}$ then the total number of edges is $\frac{1}{2} \times 16 \times 6 L^{4}=48 L^{4}$. We represent the local constraints by black squares in Fig. 11. As for the global symmetries with regards to the 2-cells, they are formed by taking the product of faces that form two-dimensional planes of which there are six, as shown in Appendix D. They are labeled by red triangles in Fig. 11.

However, not all edges, leading to the aforementioned redundancies in the stabilizers, are themselves independent. As

\footnotetext{
${ }^{9}$ Vertex operator of the vertex operator.

${ }^{10} \mathrm{An}$ equivalent description of the same statement is that if one were to take the dual of the octaplex tessellation, the 16-cell honeycomb $\{3,3,4,3\}$, then the underlying 3 -cells of the dual (corresponding to edges in the octaplex tessellation) must be tetrahedra. Given qubits are associated with edges in the dual, taking the product over faces of a tetrahedron yields identity.
} 
above, a set of 1-cells (edges) is deemed dependent if they overlap an even number of times across all neighboring 2cells. Again, the symmetries giving rise to these dependencies come in two forms: local and global. The local symmetry arises by considering the set of edges emerging from each of the vertices in the lattice. If we then consider all faces that these edges share, each face must have two of its edges in this set as by definition the chosen vertex is a vertex belonging to the face. Therefore, each vertex provides an additional constraint on the relationship between the 1-cells and there are $6 L^{4}$ such constraints, represented by black diamonds at the top of Fig. 11. The global symmetries on the 1-cells emerge from taking the product of all 1-cells (edges) forming a hyperplane, totaling four such constraints as presented in detail in Appendix E, represented by the four red squares in Fig. 11. Finally, there is one additional global symmetry for the vertices themselves each edge in the lattice is in the support of two vertices, therefore taking the product over all vertices in the lattice results in a global symmetry as their support on the edges is all even.

Therefore, the number of independent 0 -cell constraints is $6 L^{4}-1$. The number of independent 1-cell constraints is $48 L^{4}-\left(6 L^{4}-1\right)-4=42 L^{4}-3$. Finally, the number of independent 2-cell $Z$ stabilizers is $64 L^{4}-\left(42 L^{4}-3\right)-6=$ $22 L^{4}-3$. Adding the $X$ stabilizers, the total number of stabilizer generators is $2 L^{4}-1+22 L^{4}-3=24 L^{4}-4$, and thus the code has four logical qubits as hypothesized.

Finally, we would like to make a comment on the singleshot ability to perform the $Z$ stabilizer measurements. The metachecks of the 0- and 1-cells in Fig. 11 form the checks for a classical code where the bits of the code are the measurement outcomes of the $Z$ stabilizer measurements. As such, when a measurement error occurs, it causes a violation in the metachecks, allowing for its correction without having to repeat measurements, unlike the 2D toric code, for example. Given a measurement error will lead to a violation of a metacheck on its three neighboring edges, we can think of the analogy to excitations in the 2D color code where excitations come in triples where the color of an edge can be the complementary color to the color of its two neighboring endpoints. This alone should be sufficient to guarantee single-shot correction of the $Z$ stabilizer measurements, however, we have the additional layer of redundancy that is given by the 0 -cells that can identify when violations of 1-cells are misidentified, which further increases the protection against measurement errors.

\section{DISCUSSION}

We have established the existence of a 4D topological code with a transversal CCCZ gate. It is then natural to ask whether the presented techniques could be generalized to arbitrary dimension. The natural candidate in $5 \mathrm{D}$ would be to consider lattices with Schläfli symbol: $\{3,3,4, a, b\}$, where $a, b$ are integer degrees of freedom. This choice is motivated by the fact that underlying 4-cells composing the lattice are hyperoctahedra, which are the 4D analog of the octahedron. This would allow for the potential four-coloring of the vertices as required by the conditions from Sec. IIF. However, the only regular tessellation of 5D Euclidean space is from the hypercubic family: $\{4,3,3,3,4\}$, and thus no regular tessellation with the required conditions exist. Yet, there does exist a regular tessellation in hyperbolic space $(\{3,3,4,3,3\})$ which may have the desired properties and could be of independent interest as it may encode a macroscopic number of logical qubits if the correct boundary conditions can be established. It is worth pointing out again that we only consider regular lattices in this paper, where all vertices, edges, etc. are equivalent. It would be natural to conjecture that in higher dimensions breaking full symmetry within the lattice may allow for the implementation of a transversal multicontrolled- $Z$ operation by analogy to what is possible in higher-dimensional color codes [17].

It may also be of independent interest to consider hyperbolic 3D space for the existence of regular lattices that may allow for the implementation of CCZ on a growing number of logical qubits. For example, the lattice given by Schläfli symbol $\{4,3,5\}$ could be a candidate, yet establishing the correct boundary conditions, whether open or closed, remains open. One direction could be to adapt the approach taken in other hyperbolic constructions, which were studied with regard to logical memory and decoding [32], and adapt them to $3 \mathrm{D}$ hyperbolic space.

\section{ACKNOWLEDGMENTS}

The authors would like to thank Andrew Cross, Robert König, Aleksander Kubica, Michael Vasmer, and Guanyu Zhu for valuable discussions in the development of this paper. T.J.Y. is partially supported by the IBM Research Frontiers Institute.

\section{APPENDIX A: PROOF OF LOGICAL OPERATOR COMMUTATIVITY WITH STABILIZERS}

Lemma 1. The following pair of Pauli operators form a pair of anticommuting logical operators in codeblock 0 :

$$
\begin{aligned}
& \overline{\mathcal{Z}}_{\hat{w}}^{(0)}=\prod_{z \in \mathbb{Z}_{L}} Z_{\left(0,0,0, w+\frac{1}{2}\right)}^{(0)}, \\
& \overline{\mathcal{X}}_{\hat{w}}^{(0)}=\prod_{\substack{x, y, z \\
\in \mathbb{Z}_{L}}}\left[\prod_{\substack{\alpha, \beta, \gamma \\
\in\{0,1\}}} X_{\left(x, y, z, \frac{1}{2}\right)}^{(0)} X_{\left(x+\frac{1}{2}, y+\frac{1}{2}, z+\frac{1}{2}, 0\right)}^{(0)}\right. \\
&\left.\times X_{\left(x+\frac{(-1)^{\alpha}}{4}, y+\frac{(-1)^{\beta}}{4}, z+\frac{(-1)^{\gamma}}{4}, \frac{1}{4}\right)}^{(0)}\right]
\end{aligned}
$$

Proof. It is clear that the pair of Pauli operators anticommute as the only qubits they have in common is labeled by $\left(0,0,0, \frac{1}{2}\right)$. What therefore remains to be shown is that these operators do indeed commute with the stabilizers of codeblock 0 .

As discussed in the main text, the $Z$ logical operator will only intersect $X$ stabilizers of with integer coordinates $(0,0,0, w)$ which will therefore intersect the operator at two qubits: $\left(0,0,0, w \pm \frac{1}{2}\right)$, again assuming the periodicity of the 
lattice. As such, the presented $Z$ operator commutes with the stabilizers and is a logical operator.

We now show that the proposed $X$ logical operator also commutes with the $Z$ stabilizers. Suppose a $Z$ stabilizer is supported on a type-(3ii) qubit $\left(x, y, z, \frac{1}{2}\right)$ (see Sec. III B), we must show that is supported on another qubit that belong to the proposed logical $X$ operator. The only red vertices that belong to the 3-cell labeled by $\left(x, y, z, \frac{1}{2}\right)$ are $v_{r, \gamma}=(x, y, z+$ $\left.(-1)^{\gamma} \frac{1}{2}, \frac{1}{2}\right)$. In a similar manner, the only two green and blue vertices that belong to the corresponding 3-cell are $v_{g, \beta}=$ $\left(x, y+(-1)^{\beta} \frac{1}{2}, z, \frac{1}{2}\right), \quad v_{b, \alpha}=\left(x+(-1)^{\alpha} \frac{1}{2}, y, z, \frac{1}{2}\right)$. Given a triple of different color vertices, the corresponding weight-3 $Z$ stabilizer is supported on the type-(3ii) qubit and two type(3iii) qubits:

$$
\begin{aligned}
& \left\{\left(x, y, z, \frac{1}{2}\right),\right. \\
& \left(x+(-1)^{\alpha} \frac{1}{4}, y+(-1)^{\beta} \frac{1}{4}, z+(-1)^{\gamma} \frac{1}{4}, \frac{1}{4}\right), \\
& \left.\left(x+(-1)^{\alpha} \frac{1}{4}, y+(-1)^{\beta} \frac{1}{4}, z+(-1)^{\gamma} \frac{1}{4}, \frac{3}{4}\right)\right\} .
\end{aligned}
$$

As such, we have shown that any $Z$ stabilizer supported on a qubit (3ii) of the form $\left(x, y, z, \frac{1}{2}\right)$ must commute with the proposed logical $X$ operator as it has even overlap. We will argue in a similar manner for a $Z$ stabilizer supported on qubit of type (3i), $\left(x+\frac{1}{2}, y+\frac{1}{2}, z+\frac{1}{2}, 0\right)$. The vertices belonging to that 3-cell are $v_{r, \gamma}=\left\{\left(x+\frac{1}{2}, y+\frac{1}{2}, z+\right.\right.$ $\left.\left.\frac{1}{2}+(-1)^{\gamma} \frac{1}{2}, 0\right)\right\}, v_{g, \beta}=\left\{\left(x+\frac{1}{2}, y+\frac{1}{2}+(-1)^{\beta} \frac{1}{2}, z+\right.\right.$ $\left.\left.\frac{1}{2}, 0\right)\right\}, \quad v_{b, \alpha}=\left\{\left(x+\frac{1}{2}+(-1)^{\alpha} \frac{1}{2}, y+\frac{1}{2}, z+\frac{1}{2}, 0\right)\right\} . \quad$ The corresponding $Z$ stabilizer at the intersection of a given choice of three different colored vertices is

$$
\begin{aligned}
& \left\{\left(x+\frac{1}{2}, y+\frac{1}{2}, z+\frac{1}{2}, 0\right),\right. \\
& \left(x+\frac{1}{2}+(-1)^{\alpha} \frac{1}{4}, y+\frac{1}{2}+(-1)^{\beta} \frac{1}{4}, z+\frac{1}{2}+(-1)^{\gamma} \frac{1}{4},-\frac{1}{4}\right), \\
& \left.\left(x+\frac{1}{2}+(-1)^{\alpha} \frac{1}{4}, y+\frac{1}{2}+(-1)^{\beta} \frac{1}{4}, z+\frac{1}{2}+(-1)^{\gamma} \frac{1}{4}, \frac{1}{4}\right)\right\},
\end{aligned}
$$

which again has even overlap with the proposed logical $X$ operator. Thus we have shown that any $Z$ stabilizer supported on qubits of types (3i) and (3ii) in the support of the proposed logical $X$ operator must commute with the logical operator.

The only case that remains is a $Z$ stabilizer that overlaps with a qubit of type (3iii) in the proposed logical operator, but not necessarily one of type (3i) or (3ii). Suppose a $Z$ stabilizer is supported on qubit $\left(x+(-1)^{\alpha} \frac{1}{4}, y+\right.$ $\left.(-1)^{\beta} \frac{1}{4}, z+(-1)^{\gamma} \frac{1}{4}, \frac{1}{4}\right)$, then the vertices that are in the support of the associated 3-cell are $v_{r}=\{(x, y, z+$ $\left.\left.(-1)^{\gamma} \frac{1}{2}, \frac{1}{2}\right),\left(x+(-1)^{\alpha} \frac{1}{2}, y+(-1)^{\beta} \frac{1}{2}, z, 0\right)\right\}, v_{g}=\{(x, y+$ $\left.\left.(-1)^{\beta} \frac{1}{2}, z, \frac{1}{2}\right),\left(x+(-1)^{\alpha} \frac{1}{2}, y, z+(-1)^{\alpha} \frac{1}{2}, 0\right)\right\}, \quad v_{b}=$ $\left\{\left(x+(-1)^{\alpha} \frac{1}{2}, y, z, \frac{1}{2}\right),\left(x, y+(-1)^{\beta} \frac{1}{2}, z+(-1)^{\gamma} \frac{1}{2}, 0\right)\right\}$.

We already covered the cases when the $Z$ stabilizer is supported on a face with a fixed $\hat{w}$ coordinate in the set $\left\{0, \frac{1}{2}\right\}$, therefore the chosen vertices cannot all agree in the $\hat{w}$ coordinate. Without loss of generality, choose the following set of vertices: $\left\{\left(x, y, z+(-1)^{\gamma} \frac{1}{2}, \frac{1}{2}\right),(x, y+\right.$ $\left.\left.(-1)^{\beta} \frac{1}{2}, z, \frac{1}{2}\right),\left(x, y+(-1)^{\beta} \frac{1}{2}, z+(-1)^{\gamma} \frac{1}{2}, 0\right)\right\}$, which will support a $Z$ stabilizer on the following set of coordinates:

$$
\begin{aligned}
& \left\{\left(x, y+(-1)^{\beta} \frac{1}{2}, z+(-1)^{\gamma} \frac{1}{2}, \frac{1}{2}\right),\right. \\
& \left(x-\frac{1}{4}, y+(-1)^{\beta} \frac{1}{4}, z+(-1)^{\gamma} \frac{1}{4}, \frac{1}{4}\right), \\
& \left.\left(x+\frac{1}{4}, y+(-1)^{\beta} \frac{1}{4}, z+(-1)^{\gamma} \frac{1}{4}, \frac{1}{4}\right)\right\} .
\end{aligned}
$$

Note that the presented $Z$ stabilizer does indeed commute with the proposed logical operator as it intersects with two type(3iii) qubits. This will necessarily be the case for any face whose vertex coordinates do not all agree in the $\hat{w}$ coordinate.

\section{APPENDIX B: PROOF OF DISTANCE OF LOGICAL $X$ AND $Z$ OPERATORS}

Given the symmetry between the different logical operators within a codeblock as well as the different codeblocks, we will prove the distance for a single pair of logical operators $\overline{\mathcal{X}}_{\hat{w}}^{(0)}, \overline{\mathcal{Z}}_{\hat{w}}^{(0)}$.

As shown in the main section of the text, we can find many different representations of logical $\overline{\mathcal{Z}}_{\hat{w}}^{(0)}$ :

$$
\begin{aligned}
\overline{\mathcal{Z}}_{\hat{w}}^{(0)} & =\prod_{w \in \mathbb{Z}_{L}} Z_{\left(x, y, z, w+\frac{1}{2}\right)} \\
& \simeq \prod_{\substack{w \in \mathbb{Z}_{L} \\
\zeta \in \mathbb{Z}_{2}}} Z_{\left(x+\frac{(-1)^{\alpha}}{4}, y+\frac{(-1)^{\beta}}{4}, z+\frac{(-1)^{\gamma}}{4}, w+\frac{(-1)^{\zeta}}{4}\right)} \\
& =\prod_{w \in \mathbb{Z}_{L}} Z_{\left(x+\frac{(-1)^{\alpha}}{2}, y+\frac{(-1)^{\beta}}{2}, z+\frac{(-1)^{\gamma}}{2}, w\right)}, \forall x, y, z \in \mathbb{Z} .
\end{aligned}
$$

There are $2 L^{3}+(2 L)^{3}=8 L^{3}$ different nonoverlapping representations of the above logical $\bar{Z}_{w}^{(0)}$ operator. Therefore, the corresponding conjugate pair must be be supported on at least $8 L^{3}$ qubits. The following representatives have exactly that weight:

$$
\begin{aligned}
& \overline{\mathcal{X}}_{\hat{w}}^{(0)}=\prod_{\substack{x, y, z \\
\in \mathbb{Z}_{L}}}\left[\prod_{\substack{\alpha, \beta, \gamma \\
\in\{0,1\}}} X_{\left(x, y, z, \frac{1}{2}\right)}^{(0)} X_{\left(x+\frac{1}{2}, y+\frac{1}{2}, z+\frac{1}{2}, 0\right)}^{(0)}\right.
\end{aligned}
$$

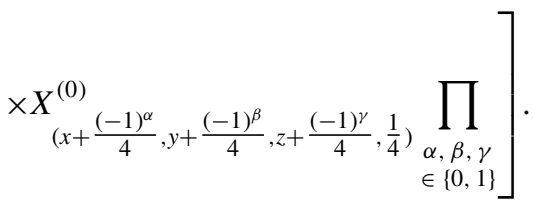

Moreover, this logical operator can be shifted in the $\hat{w}$ direction by multiplying by the appropriate set of stabilizers,

$$
\begin{aligned}
\overline{\mathcal{X}}_{\hat{w}}^{(0)}=\prod_{\substack{x, y, z \\
\in \mathbb{Z}_{L}}}\left[\prod_{\substack{\alpha, \beta, \gamma \\
\in\{0,1\}}} X_{\left(x, y, z, w+\frac{1}{2}\right)}^{(0)} X_{\left(x+\frac{1}{2}, y+\frac{1}{2}, z+\frac{1}{2}, w\right)}^{(0)}\right. \\
\\
\left.\quad \times X_{\left(x+\frac{(-1)^{\alpha}}{4}, y+\frac{(-1)^{\beta}}{4}, z+\frac{(-1)^{\gamma}}{4}, w+\frac{1}{4}\right)}^{(0)} \underset{\substack{\alpha, \beta, \gamma \\
\in\{0,1\}}}{\prod^{(0, \gamma}}\right],
\end{aligned}
$$

which gives us $L$ different disjoint representatives of the logical $\overline{\mathcal{X}}_{\hat{w}}^{(0)}$ operator, implying the $Z$ distance must be at least $L$, of which the above logical $\overline{\mathcal{Z}}_{\hat{w}}^{(0)}$ satisfy this lower bound.

Therefore, the respective $X$ and $Z$ distances of the code are $d_{X}=8 L^{3}, d_{Z}=L$.

\section{APPENDIX C: LIST OF LOGICAL PAULI OPERATORS OF 4D TORIC CODE AND ASSOCIATED CCCZ GATE}


Codeblock 0:

$$
\begin{aligned}
& \overline{\mathcal{Z}}_{\hat{x}}^{(0)}=\prod_{x \in \mathbb{Z}_{L}} Z_{\left(x+\frac{1}{2}, 0,0,0\right)}^{(0)}, \quad \overline{\mathcal{X}}_{\hat{x}}^{(0)}=\prod_{\substack{y, z, w \\
\in \mathbb{Z}_{L}}}\left[\prod_{\substack{\beta, \gamma, \zeta \\
\in\{0,1\}}} X_{\left(\frac{1}{2}, y, z, w\right)}^{(0)} X_{\left(0, y+\frac{1}{2}, z+\frac{1}{2}, w+\frac{1}{2}\right)}^{(0)} X_{\left(\frac{1}{4}, y+\frac{(-1)^{\beta}}{4}, z+\frac{(-1)^{\gamma}}{4}, w+\frac{(-1)^{\zeta}}{4}\right)}^{(0)}\right], \\
& \overline{\mathcal{Z}}_{\hat{y}}^{(0)}=\prod_{y \in \mathbb{Z}_{L}} Z_{\left(0, y+\frac{1}{2}, 0,0\right)}^{(0)}, \quad \overline{\mathcal{X}}_{\hat{y}}^{(0)}=\prod_{\substack{x, z, w \\
\in \mathbb{Z}_{L}}}\left[\prod_{\substack{\alpha, \gamma, \zeta \\
\in\{0,1\}}} X_{\left(x, \frac{1}{2}, z, w\right)}^{(0)} X_{\left(x+\frac{1}{2}, 0, z+\frac{1}{2}, w+\frac{1}{2}\right)}^{(0)} X_{\left(x+\frac{(-1)^{\alpha}}{4}, \frac{1}{4}, z+\frac{(-1)^{\gamma}}{4}, w+\frac{(-1)^{\zeta}}{4}\right)}^{(0)}\right] \text {, } \\
& \overline{\mathcal{Z}}_{\hat{z}}^{(0)}=\prod_{z \in \mathbb{Z}_{L}} Z_{\left(0,0, z+\frac{1}{2}, 0\right)}^{(0)}, \quad \overline{\mathcal{X}}_{\hat{z}}^{(0)}=\prod_{\substack{x, y, w \\
\in \mathbb{Z}_{L}}}\left[\prod_{\substack{\alpha, \beta, \zeta \\
\in\{0,1\}}} X_{\left(x, y, \frac{1}{2}, w\right)}^{(0)} X_{\left(x+\frac{1}{2}, y+\frac{1}{2}, 0, w+\frac{1}{2}\right)}^{(0)} X_{\left(x+\frac{(-1)^{\alpha}}{4}, y+\frac{(-1)^{\beta}}{4}, \frac{1}{4}, w+\frac{(-1)^{\xi}}{4}\right)}^{(0)}\right] \text {, } \\
& \overline{\mathcal{Z}}_{\hat{w}}^{(0)}=\prod_{z \in \mathbb{Z}_{L}} Z_{\left(0,0,0, w+\frac{1}{2}\right)}^{(0)}, \quad \overline{\mathcal{X}}_{\hat{w}}^{(0)}=\prod_{\substack{x, y, z \\
\in \mathbb{Z}_{L}}}\left[\prod_{\substack{\alpha, \beta, \gamma \\
\in\{0,1\}}} X_{\left(x, y, z, \frac{1}{2}\right)}^{(0)} X_{\left(x+\frac{1}{2}, y+\frac{1}{2}, z+\frac{1}{2}, 0\right)}^{(0)} X_{\left(x+\frac{(-1)^{\alpha}}{4}, y+\frac{(-1)^{\beta}}{4}, z+\frac{(-1)^{\gamma}}{4}, \frac{1}{4}\right)}^{(0)}\right] \text {. }
\end{aligned}
$$

Codeblock 1:

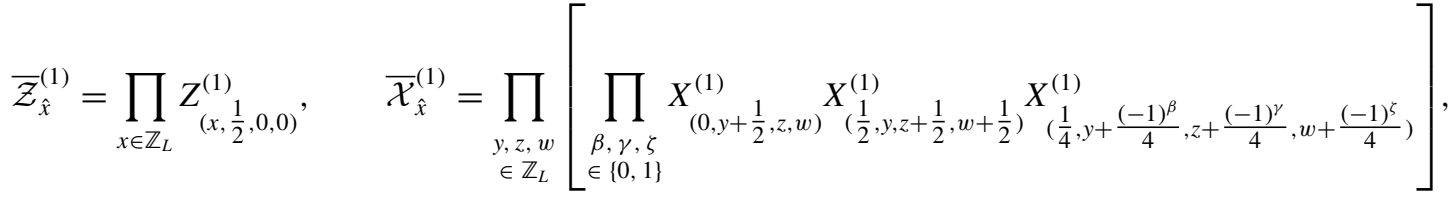

$$
\begin{aligned}
& \overline{\mathcal{Z}}_{\hat{y}}^{(1)}=\prod_{y \in \mathbb{Z}_{L}} Z_{\left(\frac{1}{2}, y, 0,0\right)}^{(1)}, \quad \overline{\mathcal{X}}_{\hat{y}}^{(1)}=\prod_{\substack{x, z, w \\
\in \mathbb{Z}_{L}}}\left[\prod_{\substack{\alpha, \gamma, \zeta \\
\in\{0,1\}}} X_{\left(x+\frac{1}{2}, 0, z, w\right)}^{(1)} X_{\left(x, \frac{1}{2}, z+\frac{1}{2}, w+\frac{1}{2}\right)}^{(1)} X_{\left(x+\frac{(-1)^{\alpha}}{4}, \frac{1}{4}, z+\frac{(-1)^{\gamma}}{4}, w+\frac{(-1)^{\xi}}{4}\right)}^{(1)}\right] \text {, }
\end{aligned}
$$

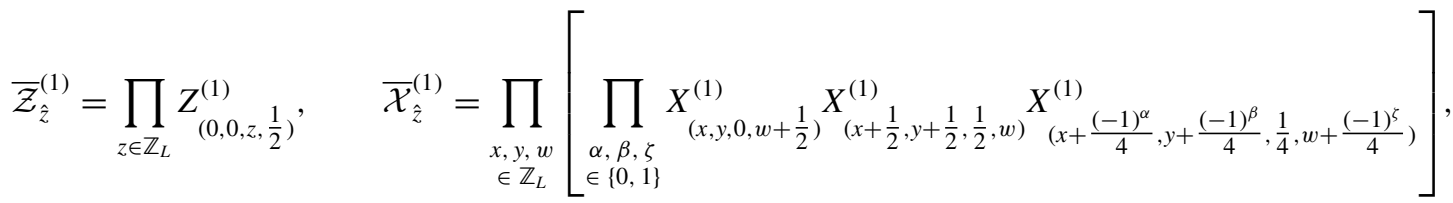

$$
\begin{aligned}
& \overline{\mathcal{Z}}_{\hat{w}}^{(1)}=\prod_{z \in \mathbb{Z}_{L}} Z_{\left(0,0, \frac{1}{2}, w\right)}^{(1)}, \quad \overline{\mathcal{X}}_{\hat{w}}^{(1)}=\prod_{\substack{x, y, z \\
\in \mathbb{Z}_{L}}}\left[\prod_{\substack{\alpha, \beta, \gamma \\
\in\{0,1\}}} X_{\left(x, y, z+\frac{1}{2}, 0\right)}^{(1)} X_{\left(x+\frac{1}{2}, y+\frac{1}{2}, z, \frac{1}{2}\right)}^{(1)} X_{\left(x+\frac{(-1)^{\alpha}}{4}, y+\frac{(-1)^{\beta}}{4}, z+\frac{(-1)^{\gamma}}{4}, \frac{1}{4}\right)}^{(1)}\right] \text {. }
\end{aligned}
$$

Codeblock 2:

$$
\begin{gathered}
\overline{\mathcal{Z}}_{\hat{x}}^{(2)}=\prod_{x \in \mathbb{Z}_{L}} Z_{\left(x, 0, \frac{1}{2}, 0\right)}^{(2)}, \quad \overline{\mathcal{X}}_{\hat{x}}^{(2)}=\prod_{\substack{y, z, w \\
\in \mathbb{Z}_{L}}}\left[\prod_{\substack{\beta, \gamma, \zeta \\
\in\{0,1\}}} X_{\left(0, y, z+\frac{1}{2}, w\right)}^{(2)} X_{\left(\frac{1}{2}, y+\frac{1}{2}, z, w+\frac{1}{2}\right)}^{(2)} X_{\left(\frac{1}{4}, y+\frac{(-1)^{\beta}}{4}, z+\frac{(-1)^{\gamma}}{4}, w+\frac{(-1)^{\zeta}}{4}\right)}^{(2)}\right], \\
\overline{\mathcal{Z}}_{\hat{y}}^{(2)}=\prod_{y \in \mathbb{Z}_{L}} Z_{\left(0, y, 0, \frac{1}{2}\right)}^{(2)}, \quad \overline{\mathcal{X}}_{\hat{y}}^{(2)}=\prod_{\substack{x, z, w \\
\in \mathbb{Z}_{L}}}\left[\prod_{\substack{\alpha, \gamma, \zeta \\
\in\{0,1\}}} X_{\left(x, 0, z, w+\frac{1}{2}\right)}^{(2)} X_{\left(x+\frac{1}{2}, \frac{1}{2}, z+\frac{1}{2}, w\right)\left(x+\frac{(-1)^{\alpha}}{4}, \frac{1}{4}, z+\frac{(-1)^{\gamma}}{4}, w+\frac{(-1)^{\zeta}}{4}\right)}^{(2)} X^{(2)}\right],
\end{gathered}
$$




$$
\begin{aligned}
& \overline{\mathcal{Z}}_{\hat{z}}^{(2)}=\prod_{z \in \mathbb{Z}_{L}} Z_{\left(\frac{1}{2}, 0, z, 0\right)}^{(2)}, \quad \overline{\mathcal{X}}_{\hat{z}}^{(2)}=\prod_{\substack{x, y, w \\
\in \mathbb{Z}_{L}}}\left[\prod_{\substack{\alpha, \beta, \zeta \\
\in\{0,1\}}} X_{\left(x+\frac{1}{2}, y, 0, w\right)}^{(2)} X_{\left(x, y+\frac{1}{2}, \frac{1}{2}, w+\frac{1}{2}\right)}^{(2)} X_{\left(x+\frac{(-1)^{\alpha}}{4}, y+\frac{(-1)^{\beta}}{4}, \frac{1}{4}, w+\frac{(-1)^{\xi}}{4}\right)}^{(2)}\right], \\
& \overline{\mathcal{Z}}_{\hat{w}}^{(2)}=\prod_{z \in \mathbb{Z}_{L}} Z_{\left(0, \frac{1}{2}, 0, w\right)}^{(0)}, \quad \overline{\mathcal{X}}_{\hat{w}}^{(2)}=\prod_{\substack{x, y, z \\
\in \mathbb{Z}_{L}}}\left[\prod_{\substack{\alpha, \beta, \gamma \\
\in\{0,1\}}} X_{\left(x, y+\frac{1}{2}, z, 0\right)}^{(2)} X_{\left(x+\frac{1}{2}, y, z+\frac{1}{2}, \frac{1}{2}\right)}^{(2)} X_{\left(x+\frac{(-1)^{\alpha}}{4}, y+\frac{(-1)^{\beta}}{4}, z+\frac{(-1)^{\gamma}}{4}, \frac{1}{4}\right)}^{(2)}\right] .
\end{aligned}
$$

Codeblock 3:

$$
\begin{aligned}
& \overline{\mathcal{Z}}_{\hat{x}}^{(3)}=\prod_{x \in \mathbb{Z}_{L}} Z_{\left(x, 0,0, \frac{1}{2}\right)}^{(3)}, \quad \overline{\mathcal{X}}_{\hat{x}}^{(3)}=\prod_{\substack{y, z, w \\
\in \mathbb{Z}_{L}}}\left[\prod_{\substack{\beta, \gamma, \zeta \\
\in\{0,1\}}} X_{\left(0, y, z, w+\frac{1}{2}\right)}^{(3)} X_{\left(\frac{1}{2}, y+\frac{1}{2}, z+\frac{1}{2}, w\right)}^{\left(X^{(3)}, y+\frac{(-1)^{\beta}}{4}, z+\frac{(-1)^{\gamma}}{4}, w+\frac{(-1)^{\xi}}{4}\right)}\right], \\
& \overline{\mathcal{Z}}_{\hat{y}}^{(3)}=\prod_{y \in \mathbb{Z}_{L}} Z_{\left(0, y, \frac{1}{2}, 0\right)}^{(3)}, \\
& \overline{\mathcal{X}}_{\hat{y}}^{(3)}=\prod_{\substack{x, z, w \\
\in \mathbb{Z}_{L}}}\left[\prod_{\substack{\alpha, \gamma, \zeta \\
\in\{0,1\}}} X_{\left(x, 0, z+\frac{1}{2}, w\right)}^{(3)} X_{\left(x+\frac{1}{2}, \frac{1}{2}, z, w+\frac{1}{2}\right)}^{(3)} X_{\left(x+\frac{(-1)^{\alpha}}{4}, \frac{1}{4}, z+\frac{(-1)^{\gamma}}{4}, w+\frac{(-1)^{\zeta}}{4}\right)}^{(3)}\right] \text {, } \\
& \overline{\mathcal{Z}}_{\hat{z}}^{(3)}=\prod_{z \in \mathbb{Z}_{L}} Z_{\left(0, \frac{1}{2}, z, 0\right)}^{(3)}, \\
& \overline{\mathcal{X}}_{\hat{z}}^{(3)}=\prod_{\substack{x, y, w \\
\in \mathbb{Z}_{L}}}\left[\prod_{\substack{\alpha, \beta, \zeta \\
\in\{0,1\}}} X_{\left(x, y+\frac{1}{2}, 0, w\right)}^{(3)} X_{\left(x+\frac{1}{2}, y, \frac{1}{2}, w+\frac{1}{2}\right)}^{(3)} X_{\left(x+\frac{(-1)^{\alpha}}{4}, y+\frac{(-1)^{\beta}}{4}, \frac{1}{4}, w+\frac{(-1)^{\xi}}{4}\right)}^{(3)}\right] \text {, } \\
& \overline{\mathcal{Z}}_{\hat{w}}^{(3)}=\prod_{z \in \mathbb{Z}_{L}} Z_{\left(\frac{1}{2}, 0,0, w\right)}^{(3)}, \\
& \overline{\mathcal{X}}_{\hat{w}}^{(3)}=\prod_{\substack{x, y, z \\
\in \mathbb{Z}_{L}}}\left[\prod_{\substack{\alpha, \beta, \gamma \\
\in\{0,1\}}} X_{\left(x+\frac{1}{2}, y, z, 0\right)}^{(3)} X_{\left(x, y+\frac{1}{2}, z+\frac{1}{2}, \frac{1}{2}\right)}^{(3)} X_{\left(x+\frac{(-1)^{\alpha}}{4}, y+\frac{(-1)^{\beta}}{4}, z+\frac{(-1)^{\gamma}}{4}, \frac{1}{4}\right)}^{(3)}\right] \text {. }
\end{aligned}
$$

The intersection of the $X$ logical operators from codeblocks $1-3$ (say, $z, y, x$ ) will result in the following $Z$ operator under $\mathrm{CCCZ}$ on codeblock 0 :

$$
\prod_{w \in \mathbb{Z}_{L}} \prod_{\zeta \in\{0,1\}} Z_{\left(0,0,0, w+\frac{1}{2}\right)}^{(0)} Z_{\left(\frac{1}{4}, \frac{1}{4}, \frac{1}{4}, w+\frac{(-1)^{\zeta}}{4}\right)}^{(0)} Z_{\left(\frac{1}{2}, \frac{1}{2}, \frac{1}{2}, w\right)}^{(0)} .
$$

Yet, when one takes the intersection of the following three $X$ stabilizers from codeblocks $1-3, v_{r}=\left(\frac{1}{2}, \frac{1}{2}, 0, w\right), v_{g}=$ $\left.\left(\frac{1}{2}, 0, \frac{1}{2}, w\right), \quad v_{b}=\left(0, \frac{1}{2}, \frac{1}{2}, w\right)\right\}$, we arrive at the following three-body $Z$ stabilizer:

$$
\left\{\left(\frac{1}{2}, \frac{1}{2}, \frac{1}{2}, w\right), \quad\left(\frac{1}{4}, \frac{1}{4}, \frac{1}{4}, w-\frac{1}{4}\right), \quad\left(\frac{1}{4}, \frac{1}{4}, \frac{1}{4}, w+\frac{1}{4}\right)\right\} .
$$

Therefore, the above logical operator is equivalent to logical $\overline{\mathcal{Z}}_{\hat{w}}^{(0)}$. Given the symmetry of the logical operators across all logical codeblocks, conditions Eqs. (4d) and (4e) are clearly satisfied when logical $X$ operators with different labels are taken across different codeblocks.

Finally, we need to show that Eq. (4c) is satisfied when $k \neq l$. Take a pair of logical $X$ operators with the different indices, say $\overline{\mathcal{X}}_{\hat{z}}^{(2)}, \overline{\mathcal{X}}_{\hat{w}}^{(3)}$. Under the action of the transversal CCCZ, $\left(\operatorname{CCCZ} \times \overline{\mathcal{X}}_{\hat{z}}^{(2)} \times \mathrm{CCCZ}^{\dagger}\right) \overline{\mathcal{X}}_{\hat{w}}^{(3)}\left(\operatorname{CCCZ} \times \overline{\mathcal{X}}_{\hat{z}}^{(2)} \times\right.$
$\left.\mathrm{CCCZ}^{\dagger}\right)^{\dagger}$, the action on their overlap will yield

$$
\begin{aligned}
\prod_{\substack{x, y \in \mathbb{Z}_{L} \\
\alpha, \beta \in \mathbb{Z}_{2}}}\left[\mathrm{CZ}_{\left(x+\frac{1}{2}, y, 0,0\right)}^{(0,1)} \mathrm{CZ}_{\left(x, y+\frac{1}{2}, \frac{1}{2}, \frac{1}{2}\right)}^{(0,1)}\right. \\
\left.\quad \times \mathrm{CZ}_{\left(x+\frac{(-1)^{\alpha}}{4}, y+\frac{(-1)^{\beta}}{4}, \frac{1}{4}, \frac{1}{4}\right)}^{(0,1)}\right] .
\end{aligned}
$$

What remains to be shown is that this operator has even overlap with the product of any $X$ stabilizers from codeblocks 0 and 1 , and is thus equivalent to logical identity. Without loss of generality, we can consider the $X$ stabilizers given by the set of coordinates: $\left(x_{0}, y_{0}, z_{0}, w_{0}\right)$ for codeblock 0 and $\left(x_{1}, y_{1}, z_{1}+\frac{1}{2}, w_{1}+\frac{1}{2}\right)$ for codeblock 1 . However, to have nonzero overlap we must have $x_{1}=x_{0}, y_{1}=y_{0}, z_{1} \in$ $\left\{z_{0}, z_{0}+1\right\}, w_{1} \in\left\{w_{0}, w_{0}+1\right\}$. Again, without loss of generality, we will choose $z_{1}=z_{0}$ and $w_{1}=w_{0}$. Therefore, the corresponding $Z$ operator above is equivalent to logical $\bar{Z}_{x}^{(3)}$. They have overlap on six 3-cells, given by coordinates

$$
\begin{aligned}
& \left\{\left(x_{0}, y_{0}, z_{0}, w_{0}+\frac{1}{2}\right), \quad\left(x_{0}, y_{0}, z_{0}+\frac{1}{2}, w_{0}\right),\right. \\
& \left(x_{0}-\frac{1}{4}, y_{0}-\frac{1}{4}, z_{0}+\frac{1}{4}, w_{0}+\frac{1}{4}\right), \\
& \left(x_{0}-\frac{1}{4}, y_{0}+\frac{1}{4}, z_{0}+\frac{1}{4}, w_{0}+\frac{1}{4}\right),
\end{aligned}
$$




$$
\begin{aligned}
& \left(x_{0}+\frac{1}{4}, y_{0}-\frac{1}{4}, z_{0}+\frac{1}{4}, w_{0}+\frac{1}{4}\right), \\
& \left.\left(x_{0}+\frac{1}{4}, y_{0}+\frac{1}{4}, z_{0}+\frac{1}{4}, w_{0}+\frac{1}{4}\right)\right\},
\end{aligned}
$$

of which it is fairly straightforward to check that this operator has even overlap with the tensor product of $\mathrm{CZ}$ operators above. Following a symmetric argument, one can show that Eq. (4c) is always satisfied for the choice of logical $X$ operators. In conclusion, the transversal CCCZ will implement a logical CCCZ, coupling logical operators in the following quartets:

$$
\begin{array}{ll}
\left\{\overline{\mathcal{X}}_{\hat{w}}^{(0)}, \overline{\mathcal{X}}_{\hat{z}}^{(1)}, \overline{\mathcal{X}}_{\hat{y}}^{(2)}, \overline{\mathcal{X}}_{\hat{x}}^{(3)}\right\}, & \left\{\overline{\mathcal{X}}_{\hat{z}}^{(0)}, \overline{\mathcal{X}}_{\hat{w}}^{(1)}, \overline{\mathcal{X}}_{\hat{x}}^{(2)}, \overline{\mathcal{X}}_{\hat{y}}^{(3)}\right\}, \\
\left\{\overline{\mathcal{X}}_{\hat{y}}^{(0)}, \overline{\mathcal{X}}_{\hat{x}}^{(1)}, \overline{\mathcal{X}}_{\hat{w}}^{(2)}, \overline{\mathcal{X}}_{\hat{z}}^{(3)}\right\}, & \left\{\overline{\mathcal{X}}_{\hat{x}}^{(0)}, \overline{\mathcal{X}}_{\hat{y}}^{(1)}, \overline{\mathcal{X}}_{\hat{z}}^{(2)}, \overline{\mathcal{X}}_{\hat{w}}^{(3)}\right\} .
\end{array}
$$

\section{APPENDIX D: GLOBAL CONSTRAINTS ON 2-CELLS}

The goal of this Appendix is to explicitly provide a global redundancy among the 2 -cells corresponding to the $Z$ stabilizers. We focus here on codeblock 0 as described in the text, yet due to the equivalence between the different codeblocks all arguments will port over to the other codeblocks as well. We will show that by taking a set of faces that form a plane, their corresponding support will cancel out and thus the set of faces will have one fewer degree of freedom.

Consider the support of the face whose vertices are given by: $\left\{\left(x, 0, \frac{1}{2}, w+\frac{1}{2}\right),\left(x, \frac{1}{2}, 0, w+\frac{1}{2}\right),\left(x, \frac{1}{2}, \frac{1}{2}, w\right)\right\}$. The support of the operator will thus be given by the following triple of qubits:

$$
\begin{aligned}
& \left(x, \frac{1}{2}, \frac{1}{2}, w+\frac{1}{2}\right), \quad\left(x-\frac{1}{4}, \frac{1}{4}, \frac{1}{4}, w+\frac{1}{4}\right), \\
& \left(x+\frac{1}{4}, \frac{1}{4}, \frac{1}{4}, w+\frac{1}{4}\right) .
\end{aligned}
$$

If we now consider face given by the following triple of vertices: $\left\{\left(x, 0, \frac{1}{2}, w+\frac{1}{2}\right),\left(x, \frac{1}{2}, 0, w+\frac{1}{2}\right),\left(x, \frac{1}{2}, \frac{1}{2}, w+\right.\right.$ $1)\}$. Then the corresponding support will be

$$
\begin{aligned}
& \left(x, \frac{1}{2}, \frac{1}{2}, w+\frac{1}{2}\right), \quad\left(x-\frac{1}{4}, \frac{1}{4}, \frac{1}{4}, w+\frac{3}{4}\right), \\
& \left(x+\frac{1}{4}, \frac{1}{4}, \frac{1}{4}, w+\frac{3}{4}\right) .
\end{aligned}
$$

Taking the product of these two operators will yield an operator with support on the following quartet:

$$
\begin{array}{ll}
\left(x-\frac{1}{4}, \frac{1}{4}, \frac{1}{4}, w+\frac{1}{4}\right), & \left(x+\frac{1}{4}, \frac{1}{4}, \frac{1}{4}, w+\frac{1}{4}\right), \\
\left(x-\frac{1}{4}, \frac{1}{4}, \frac{1}{4}, w+\frac{3}{4}\right), & \left(x+\frac{1}{4}, \frac{1}{4}, \frac{1}{4}, w+\frac{3}{4}\right) .
\end{array}
$$

Along a similar vein, the face whose vertices are $\{(x+$ $\left.\left.\frac{1}{2}, \frac{1}{2}, 0, w\right),\left(x+\frac{1}{2}, 0, \frac{1}{2}, w\right),\left(x, \frac{1}{2}, \frac{1}{2}, w\right)\right\}$ yields support:

$$
\begin{aligned}
& \left(x+\frac{1}{2}, \frac{1}{2}, \frac{1}{2}, w\right), \quad\left(x+\frac{1}{4}, \frac{1}{4}, \frac{1}{4}, w-\frac{1}{4}\right), \\
& \left(x+\frac{1}{4}, \frac{1}{4}, \frac{1}{4}, w+\frac{1}{4}\right) .
\end{aligned}
$$

Similarly, the face whose vertices are $\left\{\left(x+\frac{1}{2}, \frac{1}{2}, 0, w\right),(x+\right.$ $\left.\left.\frac{1}{2}, 0, \frac{1}{2}, w\right),\left(x+1, \frac{1}{2}, \frac{1}{2}, w\right)\right\}$ yields support:

$$
\begin{aligned}
& \left(x+\frac{1}{2}, \frac{1}{2}, \frac{1}{2}, w\right), \quad\left(x+\frac{3}{4}, \frac{1}{4}, \frac{1}{4}, w-\frac{1}{4}\right), \\
& \left(x+\frac{3}{4}, \frac{1}{4}, \frac{1}{4}, w+\frac{1}{4}\right) .
\end{aligned}
$$

Therefore, taking the product of the latter two operators will yield an operator with support:

$$
\begin{array}{ll}
\left(x+\frac{1}{4}, \frac{1}{4}, \frac{1}{4}, w-\frac{1}{4}\right), & \left(x+\frac{1}{4}, \frac{1}{4}, \frac{1}{4}, w+\frac{1}{4}\right), \\
\left(x+\frac{3}{4}, \frac{1}{4}, \frac{1}{4}, w-\frac{1}{4}\right), & \left(x+\frac{3}{4}, \frac{1}{4}, \frac{1}{4}, w+\frac{1}{4}\right) .
\end{array}
$$

Therefore, taking the plane of such weight- 4 operators by spanning all integer $x$ and $w$, the resulting operators will all cancel out and yield a global dependence. Moreover, the same argument will hold for any pair of cartesian directions, thus totally six such planar redundancies. These can be thought of analogously to the planes that form the six logical operators in the traditional 4D toric code where there is an equivalence between the $X$ and $Z$ stabilizers.

\section{APPENDIX E: GLOBAL CONSTRAINTS ON 1-CELLS}

In this Appendix, we explore the redundancy between the 1-cell constraints forming a hyperplane in the lattice. The goal of this Appendix is to provide a set of edges that share a global symmetry where all faces that emerge from these edges share have exactly two edges belonging to the set. We will consider the set of all edges between vertices $v_{0}$ and $v_{1}$ such that the $x$ coordinate of $v_{0}$ is $x_{0}=0$ and the $x$ coordinate of $v_{1}$ is $x_{1}=\frac{1}{2}$. Thus there will be three such forms of possible edges:

$$
\begin{aligned}
& \left\{\left(0, y, z+\frac{1}{2}, w+\frac{1}{2}\right),\left(\frac{1}{2}, y, z+\frac{1}{2}, w\right)\right\}, \\
& \left\{\left(0, y, z+\frac{1}{2}, w+\frac{1}{2}\right),\left(\frac{1}{2}, y, z, w+\frac{1}{2}\right)\right\}, \\
& \left\{\left(0, y+\frac{1}{2}, z, w+\frac{1}{2}\right),\left(\frac{1}{2}, y, z, w+\frac{1}{2}\right)\right\} .
\end{aligned}
$$

Without loss of generality, we shall focus on an edge between a red and green vertex point, that is, $\left\{\left(0, y, z+\frac{1}{2}, w+\frac{1}{2}\right),\left(\frac{1}{2}, y, z+\frac{1}{2}, w\right)\right\}$. Such an edge can only share faces with the following set of blue vertices: $\left(0, y-\frac{1}{2}, z+\frac{1}{2}, w\right), \quad\left(0, y+\frac{1}{2}, z+\frac{1}{2}, w\right), \quad\left(\frac{1}{2}, y, z, w+\right.$ $\left.\frac{1}{2}\right),\left(\frac{1}{2}, y, z+1, w+\frac{1}{2}\right)$, any choice of which will form an edge from the desired criteria with one of the original pair. Thus, any face sharing an edge from the criteria above will necessarily share two such edges.

Finally, given the above set of edges form a hyperplane with fixed $x_{0}=0$ and $x_{1}=\frac{1}{2}$, the choice of the $x$ coordinate here is arbitrary and can be generalized for all four Cartesian directions, thus totaling four such hyperplane constraints.

\section{APPENDIX F: BOUNDARY STABILIZERS}

In this Appendix, we explore the set of boundary stabilizers and their corresponding support. We will devote this Appendix to the case of codeblock 0 , however, similar arguments will hold for codeblocks 1-3 by symmetry. Recall we are removing all qubits that have a coordinate outside the interval $\left[\frac{1}{2}, L\right]$.

The $X$ stabilizers of codeblock 0 are given by the following sets of coordinates: $(x, y, z, w),\left(x+\frac{1}{2}, y+\frac{1}{2}, z+\right.$ $\frac{1}{2}, w+\frac{1}{2}$ ), where the $\hat{x}, \hat{y}, \hat{z}$ coordinates are constrained to be in the interval $\left[\frac{1}{2}, L\right]$ while the $\hat{w}$ coordinate is constrained to be in the interval $\left[1, L-\frac{1}{2}\right]$. We will call one of the coordinates $\hat{x}, \hat{y}, \hat{z}$ a boundary coordinate if their value is at endpoint of the allowed interval. Normally, the weight of an $X$ stabilizer in the octaplex tessellation is 24 , yet when 
boundary coordinates are present that will be changed as some of the qubits within the support of a given operator have been removed. Given a number of boundary coordinates $b$, the adjusted weight of a boundary operator is $8-b+16 / 2^{b}$. This can be derived as follows, suppose we have the following stabilizer: $\left(\frac{1}{2}, y+\frac{1}{2}, z+\frac{1}{2}, w+\frac{1}{2}\right)$, where $y, z, w$ are integers in the interval $[1, L-1]$, then the stabilizer will no longer have support on any qubit with $\hat{x}$ coordinate smaller than $\frac{1}{2}$ of which there is one with value 0 and eight with value $\frac{1}{4}$, so the total weight of the operator is $24-9=15$. Similarly, if there were two boundary coordinates (say $y=0$ above) then there would be the additional qubits that would be absent from the normal support, one $\hat{y}$ coordinate equal to 0 and the eight with value $\frac{1}{4}$ (four of which we already accounted for when the $\hat{x}$ coordinate is equal to $\frac{1}{4}$, bringing the stabilizer to: $15-1-4=10$.

There will also be modified $Z$ stabilizers on the boundary. Recall the $Z$ stabilizers defined in Sec. IIIB-IIIC. They are defined by intersection of $X$ stabilizers from the other codeblocks and have coordinates that are either: $(2 \mathrm{i})$ three quarter-integers and one half-integer, (2ii) three quarterintegers, one integer. Focusing on case (2i), the boundary $Z$ stabilizer we have to be concerned with is that where the half-integer coordinate is equal to $\frac{1}{2}$. That is, suppose we have the following $Z$ stabilizer: $\left(\frac{1}{2}, y+(-1)^{\beta} \frac{1}{4}, z+(-1)^{\alpha} \frac{1}{4}, w+\right.$ $\left.\frac{1}{2}+(-1)^{\zeta} \frac{1}{4}\right)$, for integers $y, z, w$ in the interval $[1, L-1]$, then normally the support of such a stabilizer will be on the following set of 3-cells (qubits):

$$
\begin{aligned}
& \left\{\left(\frac{1}{4}, y+(-1)^{\beta} \frac{1}{4}, z+(-1)^{\alpha} \frac{1}{4}, w+\frac{1}{2}+(-1)^{\zeta} \frac{1}{4}\right),\right. \\
& \left(\frac{3}{4}, y+(-1)^{\beta} \frac{1}{4}, z+(-1)^{\alpha} \frac{1}{4}, w+\frac{1}{2}+(-1)^{\zeta} \frac{1}{4}\right), \\
& \left.\left(\frac{1}{2}, y+(-1)^{\beta} \frac{1}{2}, z+(-1)^{\alpha} \frac{1}{2}, w+\frac{1}{2}\right)\right\} .
\end{aligned}
$$

The first such qubit has been removed, and therefore the true support of such $Z$ stabilizers will be of weight 2 . One can similarly argue for all boundary faces and show that they will be cut to only have support on a pair of qubits; this is analogous to the weight $2 Z$ stabilizers we obtain in the 3D toric code with boundaries.
[1] D. Aharonov and M. Ben-Or, Fault-tolerant quantum computation with constant error, in Proceedings of the Twenty-ninth Annual ACM Symposium on Theory of Computing (ACM, New York, NY, 1997), pp. 176-188.

[2] A. G. Fowler, M. Mariantoni, J. M. Martinis, and A. N. Cleland, Surface codes: Towards practical large-scale quantum computation, Phys. Rev. A 86, 032324 (2012).

[3] T. J. Yoder and I. H. Kim, The surface code with a twist, Quantum 1, 2 (2017).

[4] D. Litinski, A game of surface codes: Large-scale quantum computing with lattice surgery, Quantum 3, 128 (2019).

[5] C. Chamberland, G. Zhu, Theodore J. Yoder, Jared B. Hertzberg, and A. W. Cross, Topological and Subsystem Codes on Low-Degree Graphs with Flag Qubits, Phys. Rev. X 10, 011022 (2020).

[6] C. Chamberland, A. Kubica, T. J. Yoder, and G. Zhu, Triangular color codes on trivalent graphs with flag qubits, New J. Phys. 22, 023019 (2020).

[7] E. Dennis, A. Kitaev, A. Landahl, and J. Preskill, Topological quantum memory, J. Math. Phys. 43, 4452 (2002).

[8] N. Delfosse, Decoding color codes by projection onto surface codes, Phys. Rev. A 89, 012317 (2014).

[9] N. Delfosse and N. H. Nickerson, Almost-linear time decoding algorithm for topological codes, arXiv:1709.06218.

[10] A. Kubica and N. Delfosse, Efficient color code decoders in $d \geqslant 2$ dimensions from toric code decoders, arXiv:1905.07393.

[11] A. Kubica and J. Preskill, Cellular-Automaton Decoders with Provable Thresholds for Topological Codes, Phys. Rev. Lett. 123, 020501 (2019).

[12] M. Vasmer, D. E. Browne, and A. Kubica, Cellular automaton decoders for topological quantum codes with noisy measurements and beyond, Sci. Rep. 11, 2027 (2021).

[13] H. G. Katzgraber, H. Bombin, and M. A. Martin-Delgado, Error Threshold for Color Codes and Random Three-Body Ising Models, Phys. Rev. Lett. 103, 090501 (2009).
[14] H. Bombin, R. S. Andrist, M. Ohzeki, H. G. Katzgraber, and M. A. Martín-Delgado, Strong Resilience of Topological Codes to Depolarization, Phys. Rev. X 2, 021004 (2012).

[15] T. Jochym-O'Connor, A. Kubica, and T. J. Yoder, Disjointness of Stabilizer Codes and Limitations on Fault-Tolerant Logical Gates, Phys. Rev. X 8, 021047 (2018).

[16] H. Bombín and Miguel A. Martin-Delgado, Topological Quantum Distillation, Phys. Rev. Lett. 97, 180501 (2006).

[17] H. Bombín, Gauge color codes: optimal transversal gates and gauge fixing in topological stabilizer codes, New J. Phys. 17, 083002 (2015).

[18] A. Kubica and M. E. Beverland, Universal transversal gates with color codes: A simplified approach, Phys. Rev. A 91, 032330 (2015).

[19] B. Eastin and E. Knill, Restrictions on Transversal Encoded Quantum Gate Sets, Phys. Rev. Lett. 102, 110502 (2009).

[20] B. Zeng, A. W. Cross, and I. L. Chuang, Transversality versus universality for additive quantum codes, IEEE Trans. Inf, Theory 57, 6272 (2011)

[21] S. Bravyi and R. König, Classification of Topologically Protected Gates for Local Stabilizer Codes, Phys. Rev. Lett. 110, 170503 (2013).

[22] A. Kubica, B. Yoshida, and F. Pastawski, Unfolding the color code, New J. Phys. 17, 083026 (2015).

[23] M. Vasmer and D. E. Browne, Three-dimensional surface codes: Transversal gates and fault-tolerant architectures, Phys. Rev. A 100, 012312 (2019).

[24] A. R. Calderbank and P. W. Shor, Good quantum errorcorrecting codes exist, Phys. Rev. A 54, 1098 (1996).

[25] A. W. Steane, Multiple-particle interference and quantum error correction, Proc. Roy. Soc. Lond. 452, 2551 (1996).

[26] K. E. Iverson, A programming language, in Proceedings of the May 1-3, 1962, Spring Joint Computer Conference (Association for Computing Machinery, New York, 1962), pp. 345-351. 
[27] C. Vuillot and N. P. Breuckmann, Quantum pin codes, arXiv:1906.11394.

[28] H. S. M. Coxeter, Regular Polytopes (Courier Corporation, New York, 1973).

[29] M. J. G. Vasmer, Fault-Tolerant Quantum Computing with Three-Dimensional Surface Codes, Ph.D. thesis, University College, London 2019.
[30] S. B. Bravyi and A. Y. Kitaev, Quantum codes on a lattice with boundary, arXiv:quant-ph/9811052v1.

[31] E. T. Campbell, A theory of single-shot error correction for adversarial noise, Quantum Sci. Technol. 4, 025006 (2019).

[32] N. P. Breuckmann and V. Londe, Single-shot decoding of linear rate LDPC quantum codes with high performance, arXiv:2001.03568. 